\title{
Rossby Waves in Zonally Opposing Mean Flow: Behavior in Northwest Pacific Summer Monsoon
}

\author{
Hung-Chi Kuo, ${ }^{*}$ Jen-Her Chen, ${ }^{+}$R. T. Williams, and C.-P. Chang \\ Department of Meteorology, Naval Postgraduate School, Monterey, California
}

(Manuscript received 6 December 1999, in final form 27 July 2000)

\begin{abstract}
The interactions between monsoon circulations and tropical disturbances in the Northwest Pacific, where the low-level mean flow is westerly in the west and easterly in the east, are studied with a barotropic model. The authors' model results suggest that the scale contraction by the confluent background flow, the nonlinear dynamics, the $\beta$ effect, and the large-scale convergence are important for the energy and enstrophy accumulation near the region where the zonal flow reverses. The energy/enstrophy accumulation can be maintained with a continuous Rossby wave emanation upstream. The largest accumulation occurs when the emanating zonal wavelength is around $2000 \mathrm{~km}$. Longer Rossby waves experience less scale contraction and nonlinear effects while shorter Rossby waves cannot hold a coherent structure against dispersive effects.

The nonlinear energy/enstrophy accumulation mechanism is significantly different from previous linear energy accumulation theories. In the linear theories this is primarily accomplished by the slowdown of the Dopplershifted group velocity through the convergence of mean zonal advection, while in nonlinear dynamics the contraction of the zonal wave scale plays the crucial role. More importantly, after the initial energy increase by the wave accumulation, linear dynamics will lead to an eventual loss of wave energy to the mean flow due to the increase of zonal wavenumber near the critical longitude. Thus, without the presence of other forcing processes such as diabatic heating, the disturbances will decay. In nonlinear dynamics, the sharpening of the vorticity gradient as the waves approach the confluence zone leads to the development of disturbance asymmetries with respect to the central latitude. This effect is through the nonlinear interaction of Rossby waves with the planetary vorticity gradient. This development leads to a pair of vorticity centers that straddles the central latitude with the cyclone (anticyclone) in the north (south), and an elongated, weak westerly flow along the central latitude. This elongated westerly flow, which possesses a zonal wavenumber smaller than that in the linear cases, reverses the sign of the Reynold's stress and allows the energy to grow near the critical longitude, leading to intensified disturbances.

With a more realistic monsoonlike background flow, a northwestward propagation pattern with an approximately 8 -day period and 3000-km wavelength is produced, in general agreement with observed disturbances in the Northwest Pacific. The intensified disturbance may disperse energy upstream, leading to a series of trailing anticyclonic and cyclonic cells along the northwestward propagation path. When an opposing current is present, the energy dispersion leads to the formation of new disturbances in the confluence zone by vortex axisymmetrization dynamics. Thus, our results indicate that the scale contraction and nonlinear effects may cause a succession of tropical disturbances to develop without disturbance-scale diabatic effects.
\end{abstract}

\section{Introduction}

The summer monsoon in the tropical Northwest Pacific has a climatological mean trough, or depression, in the lower troposphere that has a scale of several thousand kilometers in both the zonal and meridional di-

\footnotetext{
* Permanent affiliation: Department of Atmospheric Sciences, National Taiwan University, Taipei, Taiwan.

+ Permanent affiliation: Central Weather Bureau, Taipei, Taiwan.

Corresponding author address: Dr. C. P. Chang, Department of Meteorology, Code MR/Cp, Naval Postgraduate School, Monterey, CA 93943.

E-mail: cpchang@nps.navy.mil
}

rections. On the equatorial side of this depression is an elongated belt of westerlies that meets easterlies from the central and eastern Pacific in the southeast part of the depression, which results in a confluence region in the vicinity of $140^{\circ}-160^{\circ} \mathrm{E}$. The general feature of westerlies meeting easterlies at $850 \mathrm{hPa}$ somewhere near the monsoon trough region is always present in both the synoptic and the intraseasonal timescales (e.g., Fig. 1 in Sobel and Bretherton 1999). The tropical wave-type disturbances east and west of this confluence region are observed to behave differently (Chang and Miller 1977; Takayabu and Nitta 1993). To the east, synoptic-scale waves are observed to propagate toward the west in the equatorial latitudes of $5^{\circ}-15^{\circ} \mathrm{N}$, with a period of around 4-5 days. These waves tend to be vertically tilted and 
exhibit relatively loose coupling with convection. To the west, a substantial amount of synoptic-scale variability in the Tropics is associated with deep disturbances that exhibit tight coupling with convection. During the active phase the disturbances tend to travel west/ northwestward. The growth of a new disturbance is often observed to the southeast of the previous disturbance. Chang et al. (1996) and Sobel and Bretherton (1999) presented examples of northwestwardpropagating series of synoptic-scale tropical depressions from the monsoonal confluence zone. Figure 1 contains a series of satellite images from September 1990, which show northwestward-propagating tropical cyclones and the successive formation of a new cyclone southeast of the original cyclone in the confluence zone. The 850$\mathrm{hPa}$ flow field from the Tropical Cyclone Motion 90 field experiment analysis corresponding to the satellite pictures is presented in Chang et al. (1996, their Fig. 17).

The monsoon confluence zone is an area of opposing zonal flow. Webster and Chang (1988) and Chang and Webster $(1990,1995)$ proposed that in such regions an accumulation of wave energy may occur. They used this hypothesis to explain the location and shape of extratropical teleconnection patterns, which sometimes remain constant regardless of the actual location of tropical convection. They argued that wave energy produced by tropical convection in the upper troposphere could accumulate in regions where $\partial u / \partial x$ is negative, that is, in regions of convergence of the zonal wind. These same longitudinal regions are also areas of wave energy emanation to higher latitude by Rossby wave dispersion. Holland (1995) was probably the first to point out the likely importance of linear energy accumulation for the lower-troposphere synoptic disturbances in the summertime Northwest Pacific. In a more recent study of tropical disturbances in the western Pacific, Sobel and Bretherton (1999) clearly demonstrated this possibility. Their Fig. 4 showed the overall zonal-scale compression of wave troughs and the successive northwestwardpropagating tropical cyclones from the confluence zone during August 1994. Their results suggest that the formation and maintenance of tropical cyclones may be due to the accumulation of Rossby wave activity in the convergence region of the low-level mean flow of the Northwest Pacific.

The summer monsoon region of the Northwest Pacific contains a convergence low-level mean flow with westerly winds from the west and easterly winds from the east. The zonal variation of the zonal wind is quite rapid. In this case, the linear theories of wave accumulation and vanishing group velocity are not valid in the vicinity of the critical longitude (i.e., where $C_{g}=0$ ), where the accumulation effect should be most profound. In this paper, we extended previous work by explicitly representing the nonlinear barotropic dynamics near the critical longitude, where the linear, WKB arguments used in prior studies must fail. We will study the nonlinear interactions between the Northwest Pacific summer monsoon circulations and equatorial wave disturbances. We will investigate both the mechanisms that lead to the intensification of the tropical disturbances in the confluence zone, and the processes of the successive formation of coherent tropical disturbances. The study will be carried out within the idealized framework of a $\beta$-plane nondivergent barotropic model. Although tropical disturbances are not energetically barotropic (e.g., Lau and Lau 1992), they propagate approximately as barotropic Rossby waves at low levels in most regions, including the Northwest Pacific (Sobel and Bretherton 1999), Atlantic (Shapiro 1978; Stevens 1979), and southwest Pacific (Davidson and Hendon 1989). The work by Holland (1995) and Sobel and Bretherton (1999) also suggest that diabatic processes are not needed to account for the initial development of tropical depressions in the Northwest Pacific.

Section 2 introduces the barotropic model used in the study. In section 3, the general problem of energy and enstrophy accumulation in the regions of zonal flow reversal is elucidated. In section 4 a basic flow resembling the summer monsoon circulation in the Northwest Pacific is considered. The formation of a new disturbance in the confluence zone from the southeastward Rossby energy dispersion, which is caused by a previously developed disturbance, is investigated. Concluding remarks are given in section 5.

\section{Solution methods}

We perform calculations with a nondivergent barotropic vorticity model on a double periodic domain. The nondivergent barotropic model with a basic flow can be written as

$$
\begin{aligned}
\frac{\partial \zeta^{\prime}}{\partial t}= & -\left(\frac{\partial L_{1}+N_{1}}{\partial x}+\frac{\partial L_{2}+N_{2}}{\partial y}\right)-\beta v^{\prime}-\hat{D} \zeta^{\prime} \\
& -\gamma \zeta^{\prime}-F
\end{aligned}
$$

where the linear terms is defined as

$$
\begin{aligned}
& L_{1}=\bar{u} \zeta^{\prime}+\bar{\zeta} u^{\prime}, \\
& L_{2}=\bar{v} \zeta^{\prime}+\bar{\zeta} v^{\prime},
\end{aligned}
$$

and the nonlinear terms as

$$
\begin{aligned}
& N_{1}=u^{\prime} \zeta^{\prime}, \\
& N_{2}=v^{\prime} \zeta^{\prime} .
\end{aligned}
$$

Here $u^{\prime}$ and $v^{\prime}$ are the zonal and meridional components of the perturbation velocity; $\zeta^{\prime}$ is the perturbation relative vorticity; $\bar{u}, \bar{v}$, and $\bar{\zeta}$ are the basic-state winds and basic-state relative vorticity, respectively; and $\beta$ is the planetary vorticity gradient. The dissipation timescale for the Rayleigh friction term $\gamma \zeta^{\prime}$ in (2.1) is set to be 15 days. The frictional coefficient is increased near the east-west boundary to prevent energy propa- 


\section{GMS IR 0000 UTC 1-30 September 1990}

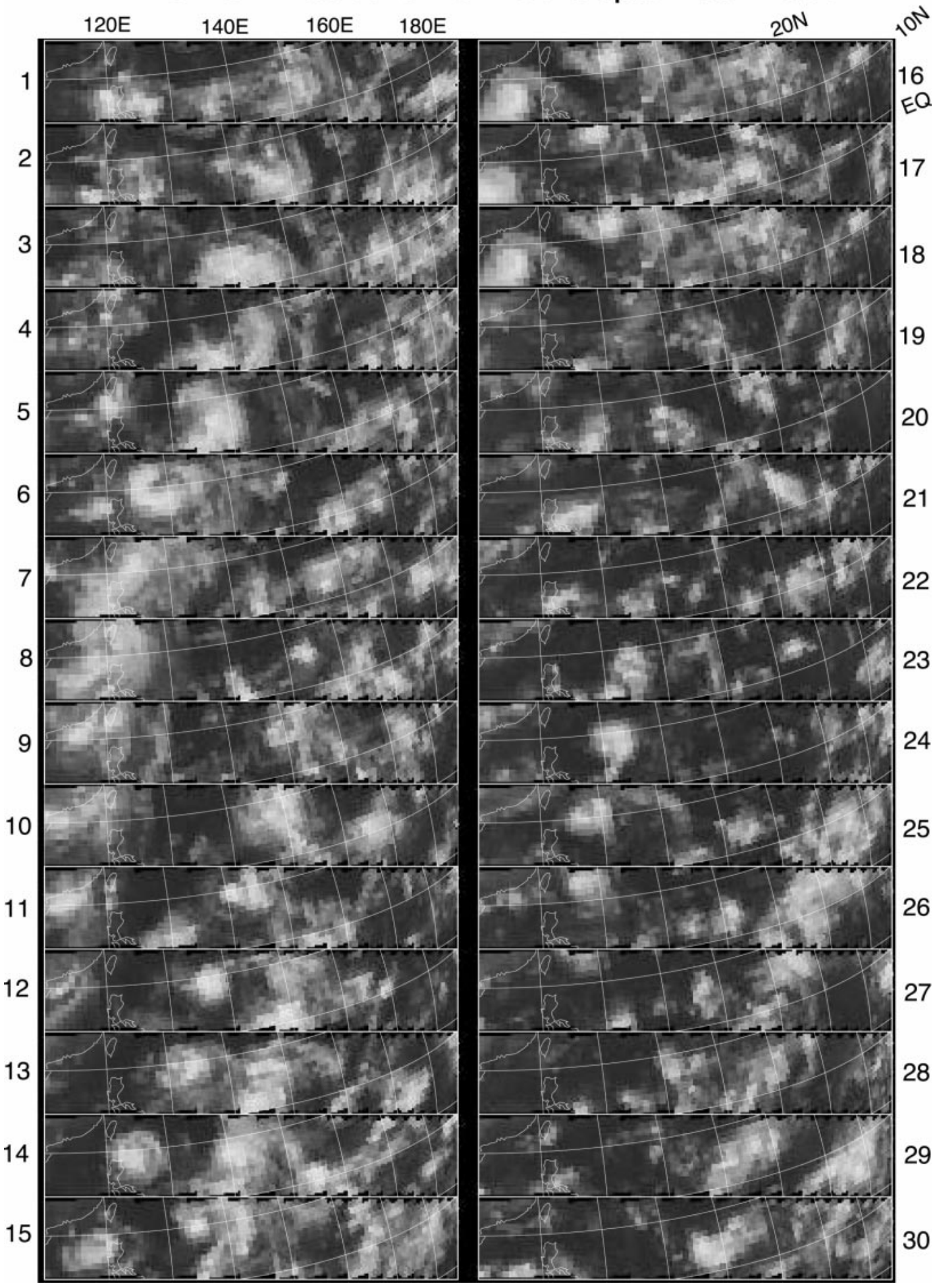

FIG. 1. Time series of GMS IR $1^{\circ} \times 1^{\circ}$ imageries in the Northwest Pacific for 0000 UTC 1-30 Sep 1990. In each day the latitude lines (oriented lower left to upper right) shown are, from left to right, $20^{\circ} \mathrm{N}, 10^{\circ} \mathrm{N}$, and equator. The corresponding 850-hPa flow field is shown in Chang et al. (1996, their Fig. 17). 
gation out of one side of domain from coming back at the other. The term $-\hat{D} \zeta^{\prime}$ represents the convergence forcing of $\zeta^{\prime}$ by the large-scale convection. The forcing term $F$ in (2.1) represents a Rossby wave maker that introduces waves along the eastern boundary with maximum amplitude at some central latitude. The Rossby wave forcing varies sinusoidally on the boundary with a period in accordance with the zonal and meridional wavenumbers and the background flow. This forcing function is defined as

$$
F=A \cos ^{2}\left[l\left(y-y_{0}\right)\right] \sin (\omega t) \exp \left(-\frac{\left|x-x_{0}\right|}{x_{d}}\right) \text {. }
$$

The wave forcing is centered on $\left(x_{0}, y_{0}\right)$ and set to zero for $\left|y-y_{0}\right| \geq \pi /(2 l)$ in the domain. The constant $x_{0}$ is set to be at the eastern edge of the computation domain as the constant $y_{0}$ is set to be in the middle of the domain. The constant $x_{d}$ is the $e$-folding width in the $x$ direction for the wave-forcing function, and $l$ is the meridional wavenumber. The meridional wavelength of westward Rossby waves is set to be $1200 \mathrm{~km}$ while the zonal wavelength is varied in the numerical experiments. In all but one experiment in which we use a $1200-\mathrm{km}$ zonal wavelength (e.g., in Fig. 6), the zonal wavelengths chosen are greater than the meridional wavelength. With a basic flow $\bar{u}$, we can adjust the zonal wavelengths to yield different frequencies according to the Rossby wave dispersion relationship. For example, the emanating Rossby waves have a timescale of 3 days when a zonal wavelength of $2500 \mathrm{~km}$ and a $\bar{u}$ of $7.8 \mathrm{~m} \mathrm{~s}^{-1}$ are used. Unless otherwise specified, the wave amplitude $A$ used in the paper is $0.75 \times 10^{-5} \mathrm{~s}^{-1}$, which is a typical observbed $\zeta^{\prime}$ amplitude in the region. A rectangular domain of $24000 \mathrm{~km}$ by $12000 \mathrm{~km}$ with a resolution of $100 \mathrm{~km}$ is used for the numerical experiments. The discretization of the barotropic model is based on the Fourier spectral method in space and the leapfrog method in time. The coefficient of the Robert filter in the time integration is 0.015 . Because the perturbation velocity is nondivergent, there is no difference between the equatorial $\beta$ plane and the midlatitude $\beta$ plane in our calculations. Our central latitude does not have to be the equator. We perform simulations based on both the linear and nonlinear models. The linear model uses (2.1) without the nonlinear $N_{1}$ and $N_{2}$ terms. The behavior of the solution will be studied for selected monsoonlike flows.

\section{Tropical disturbance development by wave accumulation}

Two types of background flows are considered in our numerical experiments. The first type involves a background flow with opposing zonal currents and no meridional current. The basic zonal flow is defined as

$$
\bar{u}=-\frac{2 u_{0}}{L}\left(x-\frac{L}{2}\right), \quad 0 \leq x \leq L,
$$

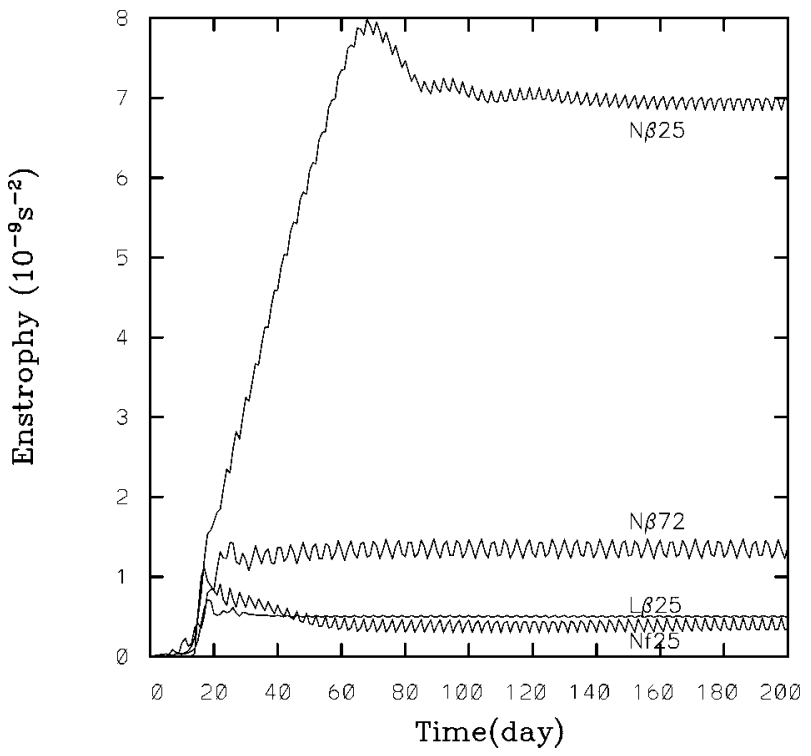

FIG. 2. The enstrophy as a function of time for the opposing background flow experiments. The four experiments are a nonlinear $\beta$-plane calculation with a forcing zonal wavelength of $2500 \mathrm{~km}(\mathrm{~N} \beta 25)$, a nonlinear $f$-plane calculation with a forcing zonal wavelength of $2500 \mathrm{~km}$ (Nf25), a linear $\beta$-plane calculation with a forcing zonal wavelength of $2500 \mathrm{~km}(\mathrm{~L} \beta 25)$, and a nonlinear $\beta$-plane calculation with a forcing zonal wavelength of $7200 \mathrm{~km}(\mathrm{~N} \beta 72)$.

where $L=24000 \mathrm{~km}$ and $u_{0}=7.8 \mathrm{~m} \mathrm{~s}^{-1}$. The associated mean flow convergence (i.e., $d \bar{u} / d x$ ) is $-2 u_{0} / L$, which has a value of $0.7 \times 10^{-6} \mathrm{~s}^{-1}$. Note that the characteristic spatial scale as estimated by $[(d u / d x) / u]^{-1}$ varies linearly away from the critical longitude (i.e., $x$ $=L / 2$ and $\bar{u}=0)$. The second type of background flow considered is a nondivergent deformation flow. This flow uses the zonal wind in (3.1) and a meridional wind defined as

$$
\bar{v}=\frac{2 u_{0}}{L}\left(y-\frac{L}{2}\right), \quad 0 \leq y \leq \frac{L}{2} .
$$

Note that the mean vorticity $\bar{\zeta}$ is zero for both the opposing and the deformation basic flows.

In the first type of experiment the opposing background flow provides not only a zone of confluence near the critical longitude but also a zone of convergence (i.e., $d \bar{u} / d x<0$ ). The motivation for this choice of basic flow is to emphasize the flow characteristics in the summer Northwest Pacific where the low-level flow shows rapid zonal variation and reversal.

Figure 2 shows the enstrophy as a function of time for the opposing flow experiments. The four experiments are nonlinear $\beta$-plane calculation with a forcing zonal wavelength of $2500 \mathrm{~km}$, nonlinear $f$-plane calculation with a forcing zonal wavelength of $2500 \mathrm{~km}$, linear $\beta$-plane calculation with a forcing zonal wavelength of $2500 \mathrm{~km}$, and nonlinear $\beta$-plane calculation with a forcing zonal wavelength of $7200 \mathrm{~km}$. The $f$-plane experiment is carried out by setting $\beta$ to zero 
(a) Kinetic Energy $\left(10^{-1} \mathrm{~m}^{2} \mathrm{~s}^{-2}\right)$

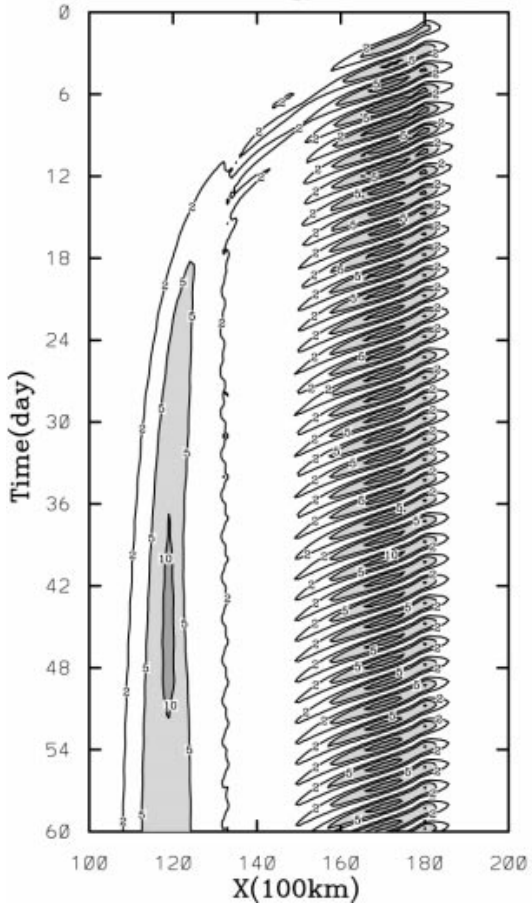

(b) Kinetic Energy $\left(10^{-1} \mathrm{~m}^{2} \mathrm{~s}^{-2}\right)$

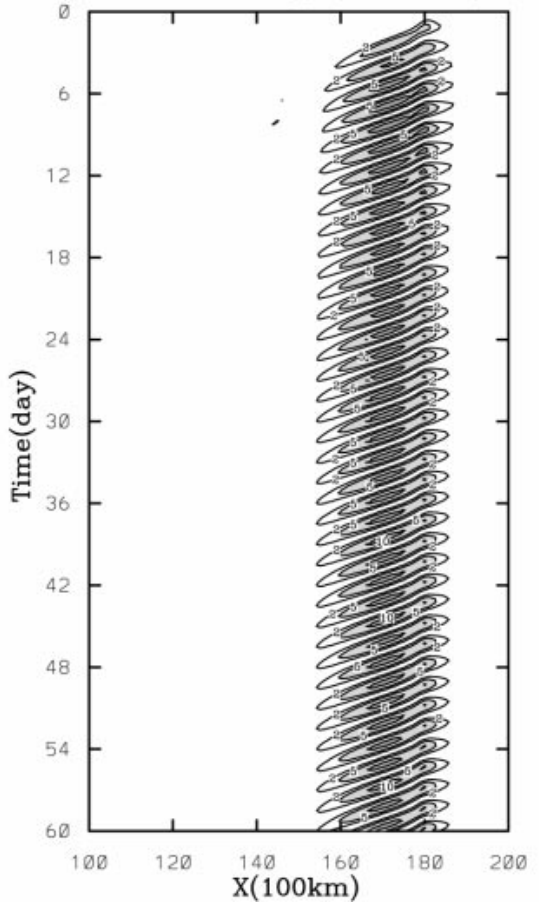

(c) Kinetic Energy $\left(10^{-1} \mathrm{~m}^{2} \mathrm{~s}^{-2}\right)$

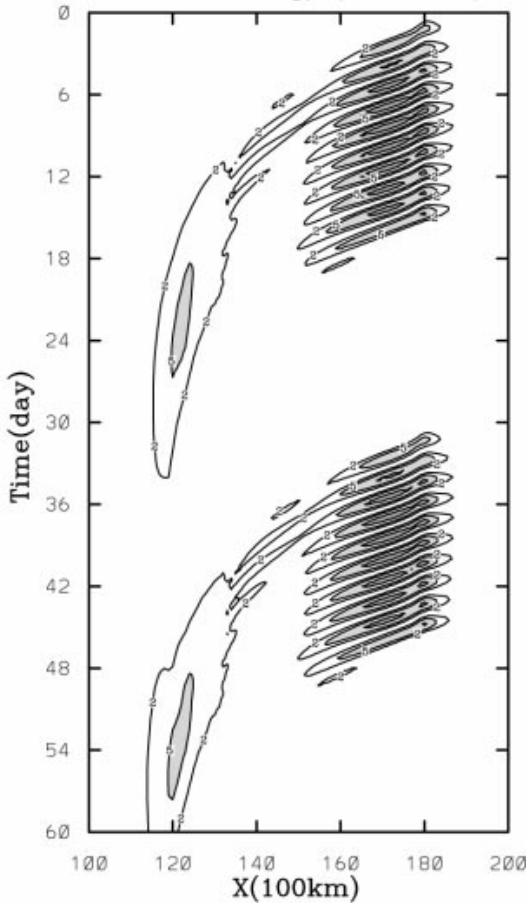

FIG. 3. Hovmöller diagram of kinetic energy for (a) nonlinear $\beta$-plane calculation with a forcing zonal wavelength $2500 \mathrm{~km}$, (b) linear $\beta$-plane calculation with a forcing zonal wavelength $2500 \mathrm{~km}$, and (c) nonlinear $\beta$-plane calculation with a forcing zonal wavelength 2500 $\mathrm{km}$ and the wave forcing turned off at day 15 and turned on again at day 30 .

in (2.1). The $f$-plane calculation is included here as a reference for the $\beta$-plane experiments. The enstrophy is averaged in the region containing the confluence zone near the critical longitude (i.e., $11500 \mathrm{~km} \leq x \leq 12500$ $\mathrm{km}$ and $0 \mathrm{~km} \leq y \leq 12000 \mathrm{~km}$ ). The $1000-\mathrm{km}$ region containing critical longitude is chosen because we are interested in the formation of hundreds-kilometers spatial-scale tropical-depression-type disturbances.

In agreement with the linear analysis of Farrel and Watterson (1985), the linear solutions did not show much enstrophy accumulation in the zone where the zonal flow reverses. On the other hand, the nonlinear $\beta$ calculation with a forcing zonal wavelength of $2500 \mathrm{~km}$ shows an enstrophy accumulation in the confluence zone. This accumulation cannot be accounted for directly by the mean convergence, as the specified laregescale mean flow convergence associated with the mean flow would take about 100 days to change the vorticity from $10^{-5} \mathrm{~s}^{-1}$ to $10^{-4} \mathrm{~s}^{-1}$ (enstrophy $10^{-8} \mathrm{~s}^{-2}$ ). Moreover, the convergence field gives an $e$-folding timescale approximately the same as the Rayleigh damping.

Figure 3 is a Hovmöller diagram of the kinetic energy averaged in the confluence zone for (a) nonlinear $\beta$-plane calculation with a forcing zonal wavelength 2500 $\mathrm{km}$, (b) linear $\beta$-plane calculation with a forcing zonal wavelength $2500 \mathrm{~km}$, and (c) nonlinear $\beta$-plane calculation with a forcing zonal wavelength $2500 \mathrm{~km}$ and with the wave forcing turned off at day 15 and turned on again at day 30. The results in Fig. 3 are in agreement with Fig. 2 in that the kinetic energy is accumulated in the nonlinear $\beta$-plane calculation with a forcing zonal wavelength of $2500 \mathrm{~km}$, but not in the linear case. In addition, Fig. 3c suggests that the energy and enstrophy accumulation is maintained only in the case with a continuous Rossby wave emanation from the eastern boundary. Without the continuous wave emanation, the accumulated energy is dissipated in 15 days by the Rayleigh friction.

Figure 3 also indicates an energy growth and decay pattern in all three cases. From east to west, the wave energy increases from $x=18000 \mathrm{~km}$ to $x=17000$ $\mathrm{km}$ but decreases from $x=17000 \mathrm{~km}$ to $x=16000$ $\mathrm{km}$. In addition, there is a gap in the energy from $x=$ $16000 \mathrm{~km}$ to $x=13000 \mathrm{~km}$ in the nonlinear cases (Figs. 3a and 3c). The energy pattern may be explained by the energy equation for barotropic Rossby waves in opposing zonal currents (Farrel and Watterson 1985),

$$
\frac{\partial E}{\partial t}+\frac{\partial}{\partial x}\left(C_{g} E\right)=E \bar{u}_{x} \frac{k^{2}-l^{2}}{k^{2}+l^{2}},
$$

where $E$ is the wave energy; $k$ and $l$ are the zonal and meridional wavenumbers, respectively; and $C_{g}=\bar{u}+$ $\beta\left(k^{2}-l^{2}\right) /\left(k^{2}+l^{2}\right)^{2}$ is the Doppler-shifted group velocity. The initial energy increase from $x=18000 \mathrm{~km}$ to $x=17000 \mathrm{~km}$ region is due to a slowdown of the Doppler-shifted group velocity $\left(\partial C_{g} E / \partial x<0\right)$. The two contributors to the slowdown of $C_{g}$ are the convergence 
(a)

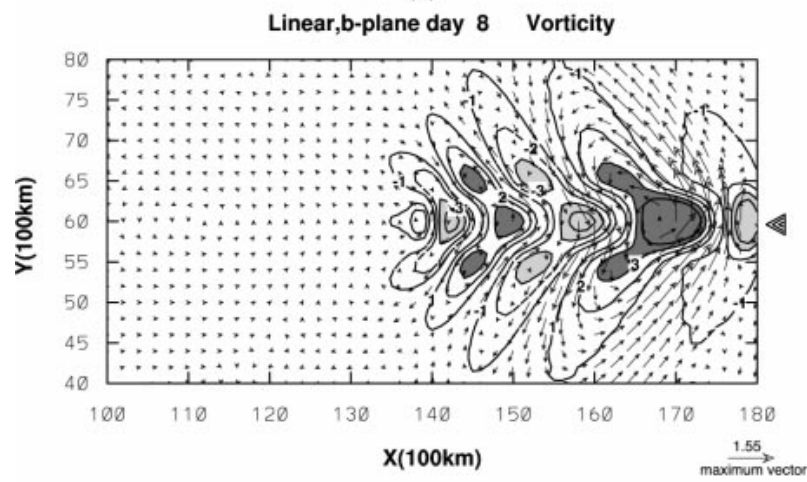

(b)

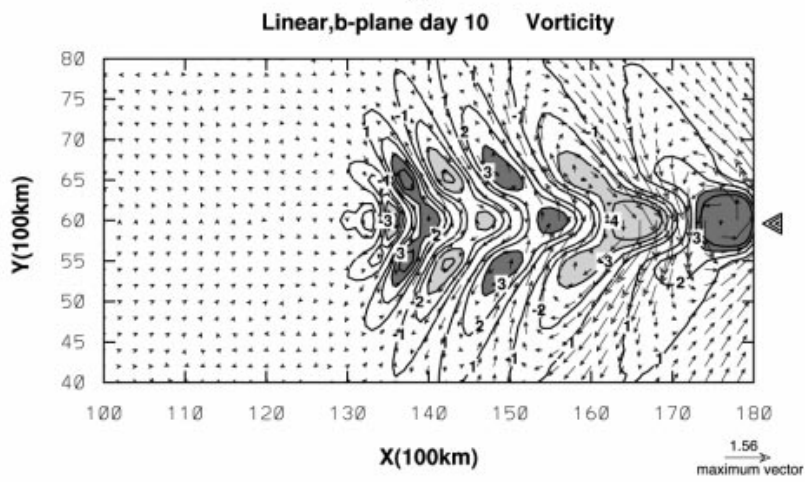

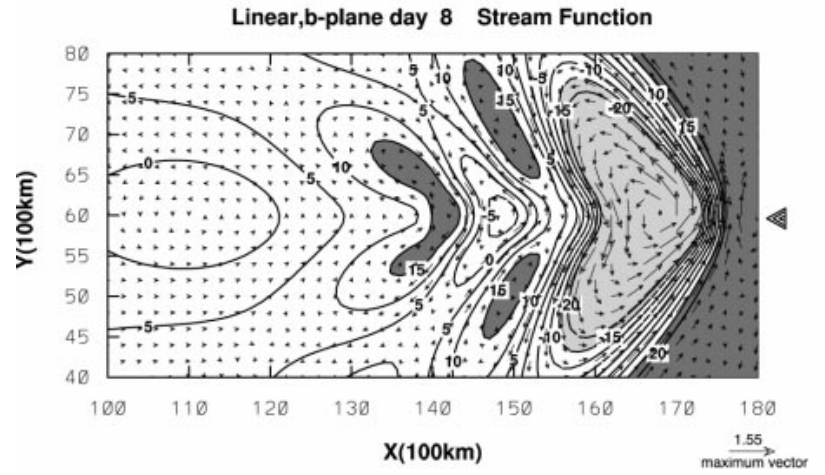

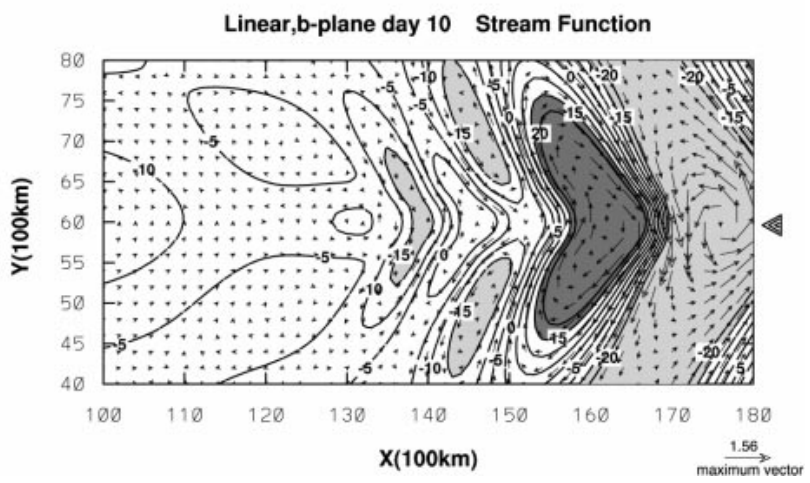

FIG. 4. The wind vectors and the vorticity and streamfunction fields for the linear $\beta$-plane calculation with a forcing zonal wavelength $2500 \mathrm{~km}(\mathrm{~L} \beta 25)$ at (a) day 8, (b) day 10, and (c) day 20. The vorticity isopleth interval is $10^{-6} \mathrm{~s}^{-1}$, with values $>3 \times 10^{-6} \mathrm{~s}^{-1}$ heavyshaded and values $<-3 \times 10^{-6} \mathrm{~s}^{-1}$ light-shaded. The streamfunction isopleth interval is $5 \times 10^{4} \mathrm{~m}^{2} \mathrm{~s}^{-1}$, with values $>15 \times 10^{4} \mathrm{~m}^{2} \mathrm{~s}^{-1}$ heavy-shaded and values $<-15 \times 10^{4} \mathrm{~m}^{2} \mathrm{~s}^{-1}$ light-shaded. An arrow on the right edge of the diagram indicates the central latitude $(Y=$ $6000 \mathrm{~km}$ ) of the eastern boundary wave forcing.

of the mean flow and the scale contraction (the confluence effect) of the zonal wave scale. The scale contraction increases the wavenumber $k$ and thus slows down the eastward intrinsic group velocity $\beta\left(k^{2}-l^{2}\right) /$ $\left(k^{2}+l^{2}\right)^{2}$. In addition to the convergence of group velocity, the energy is also gained via the Reynold's stress term on the right-hand side of (3.3) as long as $l$ $>k$, since $\bar{u}_{x}<0$. The kinetic energy Hovmöller diagram for nonlinear $f$-plane calculation is very similar to the linear $\beta$-plane calculation (i.e., Fig. $3 b$ ), and thus it is not shown here. Since the $f$-plane supports no Rossby wave propagation, the local energy increase in $f$-plane calculation can be due only to the mean flow convergence (i.e., slowdown of the zonal advection). The similarity in the Hovmöller diagrams between the $f$-plane calculation and the linear $\beta$-plane calculation suggests that the dominate contributor to the convergence of $C_{g} E$ is through the convergence of mean zonal advection. It follows that the convergence effect, rather than the confluence effect, is required to get wave energy accumulation from $x=18000 \mathrm{~km}$ to $x=17000$ $\mathrm{km}$. On the other hand, the energy decrease from $x=$ $17000 \mathrm{~km}$ to $x=16000 \mathrm{~km}$ is related to the confluencescale reduction. As discussed in Farrel and Watterson
(1985), the initial increase of $E$ through the accumulation and the increase of zonal wavenumber $k$ through the scale contraction result in eventual wave energy loss by the Reynold's stress term on the right-hand side of (3.3) (i.e., $E$ is large, $k^{2}-l^{2}>0$ and $\bar{u}_{x}<0$ ). The characteristic scale of the mean flow varies considerably near the critical longitude, and the applicability of the WKB approximation for $C_{g}$ break down. The breakdown of the linear theory is also noticed in Sobel and Bretherton (1999). In their Fig. 7, no calculations of ray tracing are performed in the $1000-\mathrm{km}$ region containing the critical longitude. The energy accumulation near the critical longitude in Figs. 3a and $3 \mathrm{c}$ can be explained only by the nonlinear processes.

The difference between the linear and nonlinear dynamics of energy and enstrophy accumulation can be studied by comparing their vorticity and streamfunction fields. These fields within an $8000 \mathrm{~km} \times 4000 \mathrm{~km}$ rectangular region of the computational domain are shown in Figs. 4 and 5. Figure 4 displays the vorticity and streamfunction fields from the linear $\beta$-plane calculation with a forcing zonal wavelength of $2500 \mathrm{~km}$ at (a) day 8 , (b) day 10 , and (c) day 20 . We observe energy radiating out from the central latitude by the Rossby wave 
(c)
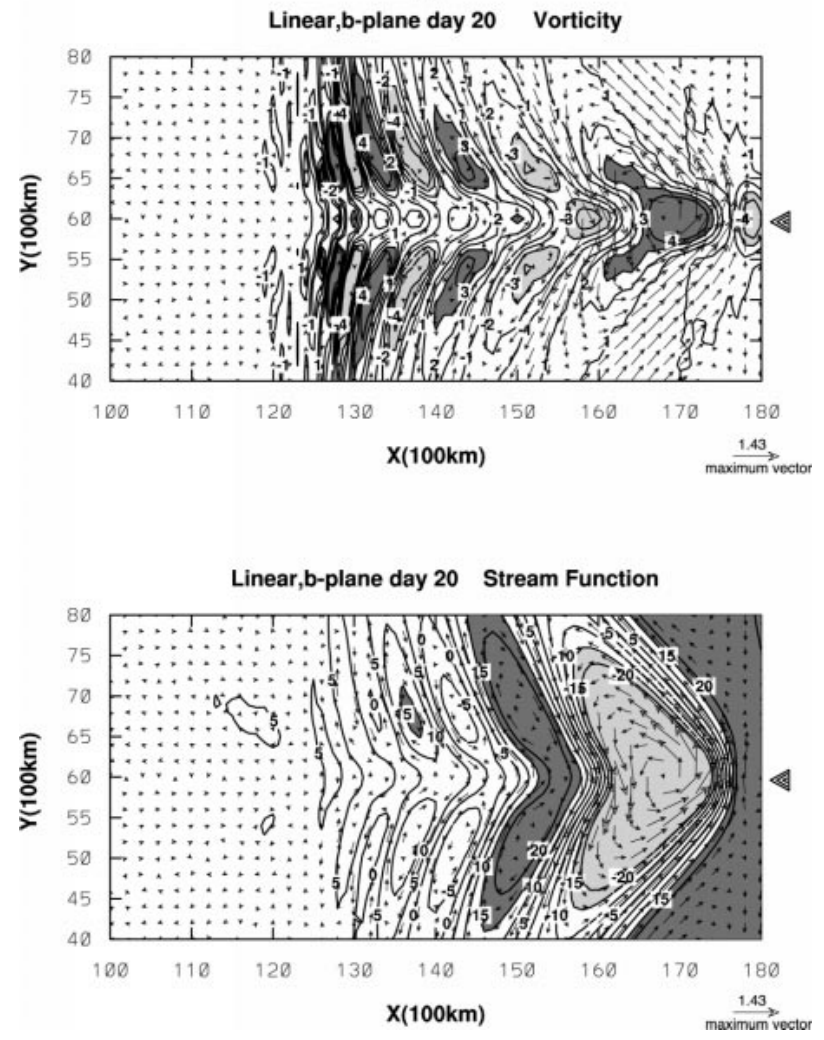

FIG. 4. (Continued)

energy dispersion process. Our results do not require the presence of a trapping basic-state flow and or modal Rossby wave forcing. This energy dispersion and leakage do not occur in our $f$-plane calculations (not shown). In general, the amplitude of the waves in the $\beta$-plane calculations decreases somewhat due to the energy dispersion. The scale contraction of the waves by the background flow can be seen as the waves approach the confluence region. Note that the plane waves used in the opposing zonal currents analysis by Farrell and Watterson (1985) allow no energy leakage. All the fields are symmetric with respect to the central latitude (i.e., $y=6000 \mathrm{~km})$ in the linear calculation. Figure 5 is similar to Fig. 4, but for the nonlinear $\beta$-plane calculation at (a) day 8, (b) day 10, (c) day 20, and (d) day 30. A conspicuous difference from Fig. 4 is the disturbance asymmetries with respect to the central latitude, which become more pronounced as the waves approach the confluence region. As the zonal scale of the incoming waves decreases when the waves approach the critical longitude (i.e., $\bar{u}=0$ at $x=12000 \mathrm{~km}$ ), the scale contraction causes a tightening of the vorticity gradient in the waves. Nonlinear effects become important as a result of the vorticity gradient sharpening (e.g., Dritschel 1998). In addition to advection by the mean flow, the cyclone (anticyclonic) circulations drift northwestward (southeastward) due to the nonlinear interaction of the vorticity fields with the planetary background flow (e.g., Chan and Williams 1987). In the presence of the mean easterly background flow, the westward drift speed is different for the cyclonic and anticyclonic circulations. The nonlinear effects induce finite-scale asymmetries across the central latitude. The asymmetries eventually result in a pair of vorticity centers, which straddle the central latitude near the critical longitude. The cyclone (anticyclone) is north (south) of the central latitude. This vorticity pair results in an elongated westerly flow along the central latitude. The westerly, which is produced within 10 days, has a zonal scale of about $3000 \mathrm{~km}$ and a speed of about $1 \mathrm{~m} \mathrm{~s}^{-1}$. The elongated westerlies, which possess a much smaller zonal wavenumber as compared to that in the linear cases, reverse the sign of the Reynold's stress in (3.3) and allow the energy to cumulate near the critical longitude. Figures $5 \mathrm{c}$ and $5 \mathrm{~d}$ indicate that the energy accumulation at the critical longitude is accompanied with this westerly anomaly production. Figure 5 is in general agreement with the simulation of Webster and Chang (1988, their Fig. 21). They also showed that a pair of vorticity centers with a zonal scale of about $3000 \mathrm{~km}$ straddle the equator in the confluence/convergence zone. It is interesting to note that in Fig. 5 some waves appear to have penetrated the critical longitude in our nonlinear calculation, while all linear waves (Fig. 4) disappear before reaching the critical longitude.

To study the effect of the emanating wave on the enstrophy accumulation, we calculate the averaged enstrophy from day 140 to day 200 as a function of the forced zonal wavelength. Figure 6 indicates that wavelengths around $2000 \mathrm{~km}$ are favored for larger enstrophy accumulation in the nonlinear $\beta$-plane experiments. It appears that there will be less scale contraction and nonlinear effects for westward Rossby waves with zonal wavelength longer than $3000 \mathrm{~km}$. On the other hand, shorter Rossby waves with a zonal wavelength less than $2000 \mathrm{~km}$, without diabatic effects on the disturbance scale, cannot hold themselves in a coherent manner against the dispersive effects. Thus, the reasons for the more significant enstrophy accumulation around zonal wavelength $2000 \mathrm{~km}$ are the proper scale contraction by background flow plus the nonlinear dynamics on the $\beta$ plane.

To further test the idea of nonlinear effects, we carry out several experiments with different forcing wave amplitudes. Figure 7 shows the averaged enstrophy from day 140 to day 200 as a function of forcing wave amplitude squared. The three curves are the nonlinear $\beta$-plane calculation with a forcing zonal wavelength $2500 \mathrm{~km}$, the nonlinear $\beta$-plane calculation with a forcing zonal wavelength $7200 \mathrm{~km}$, and the linear $\beta$-plane calculation with a forcing zonal wavelength $2500 \mathrm{~km}$. The change of averaged enstrophy as a function of amplitude squared of incoming waves for the nonlinear $\beta$-plane calculations is nonlinear in nature. Figure 7 supports the idea that the insignificant enstrophy ac- 
(a)
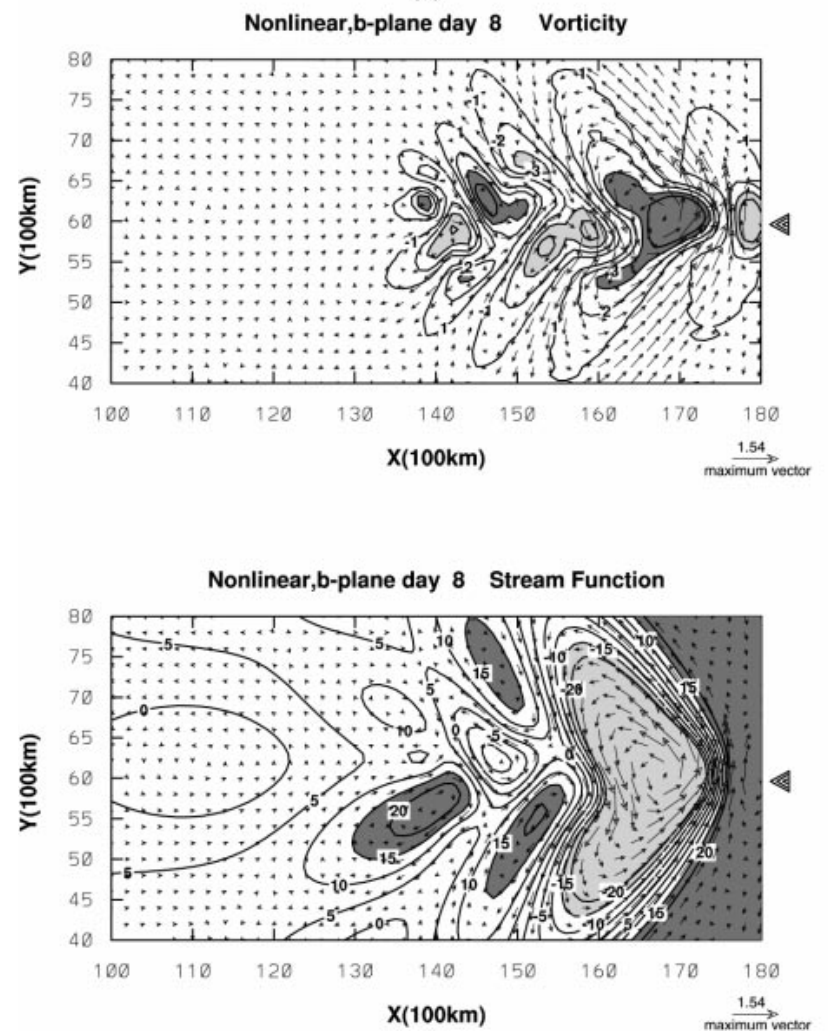

(b)
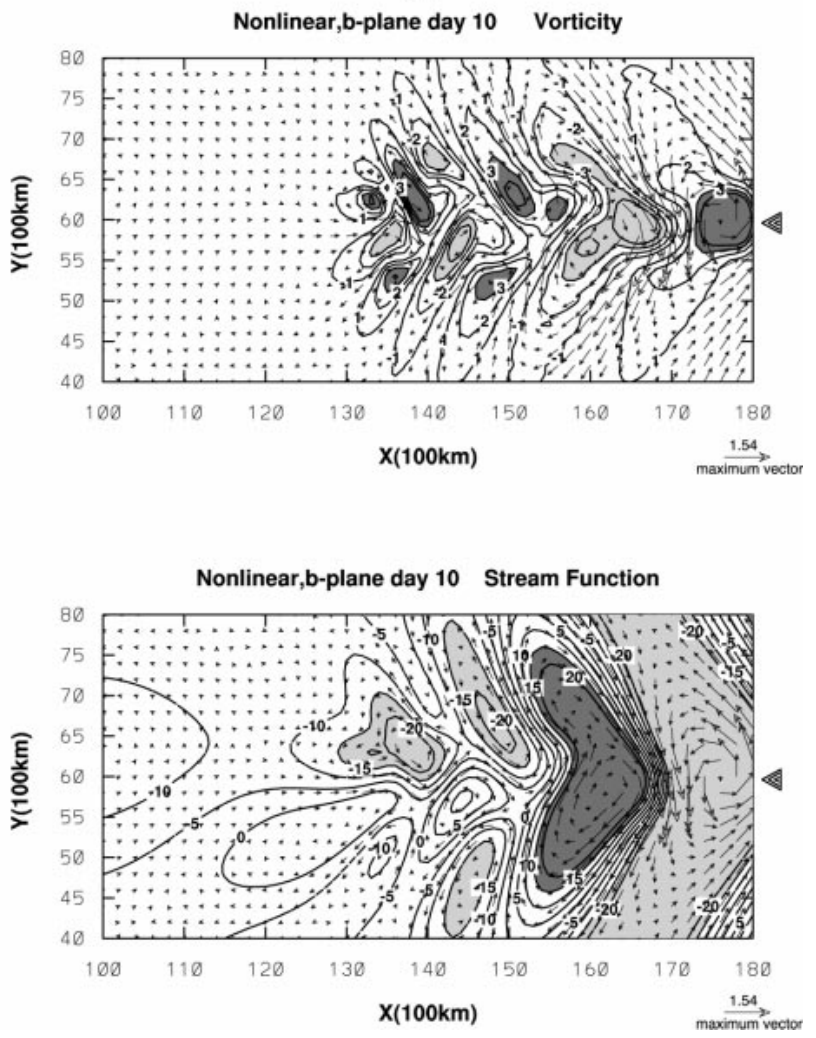

FIG. 5. Same as in Fig. 4 except for the nonlinear $\beta$-plane calculation with a forcing zonal wavelength $2500 \mathrm{~km}(\mathrm{~N} \beta 25)$ at (a) day 8 , (b) day 10 , (c) day 20, and (d) day 30 .

cumulation in the long emanating zonal wavelength $(7200 \mathrm{~km})$ probably results from an insufficient scale contraction and a weaker nonlinear effect. The nonlinear effect is clearly significant in the experiment with a wavelength of $2500 \mathrm{~km}$.

The basic-state opposing flows contain regions of both confluence and convergence. The effect of scale contraction in the confluence region and that of the wave accumulation by convergence may be separated by considering the second type experiments with the deformation basic flow defined by (3.1) and (3.2). This deformation flow allows a confluence effect near the critical longitude, but no convergence effect (i.e., $d \bar{u} / d x+$ $d \bar{v} / d y=0$ ). Any large-scale convergence (i.e., $\hat{D} \zeta^{\prime}$ ) has to be added. Figure 8 is similar to Fig. 2 except for the deformation background flow experiments. The three curves show the nonlinear $\beta$-plane calculation with an added convergence, the linear $\beta$-plane calculation with an added convergence, and the nonlinear $\beta$-plane calculation without added convergence. The forcing zonal wavelength is $2500 \mathrm{~km}$ for all cases. The added convergence has a Gaussian profile centered at $x=12000$ $\mathrm{km}$ and $y=6000 \mathrm{~km}$ with a $2500 \mathrm{~km} e$-folding distance in both horizontal directions. The peak magnitude of convergence is $-4 \times 10^{-6} \mathrm{~s}^{-1}$. In the absence of the added convergence, we observe from Fig. 8 that ac- cumulation in the nonlinear $\beta$-plane calculation is insignificant in the deformation basic flow case. Thus, the added convergence is important for energy and enstrophy accumulations. This is because the added $\hat{D}$ here assumes the role of the convergence of zonal mean advection in the previous zonal opposing currents experiments. Without this convergence in the background flow, it will be difficult for the initial upstream accumulation to occur. The large-scale convergence may be interpreted as coming from the large-scale monsoon convection, which is different from the disturbancescale convergence effect.

\section{Energy dispersion in a monsoonlike basic flow}

We now consider the energy dispersion in a monsoonlike background flow. Figure 9 shows the wind vectors in a monsoonlike background flow with contours of (a) wind speed and (b) velocity potential. The monsoonlike flow is constructed by adding a convergence zone (as indicated by the velocity potential field in Fig. $9 \mathrm{~b})$ to the basic deformation flow. The maximum convergence is $-4 \times 10^{-6} \mathrm{~s}^{-1}$, which is about the same as the averaged peak convergence value at $850 \mathrm{hPa}$ in the Northwest Pacific during summer from 1986 to 1995. This basic flow is southeasterly in the northwestern part 
(c)

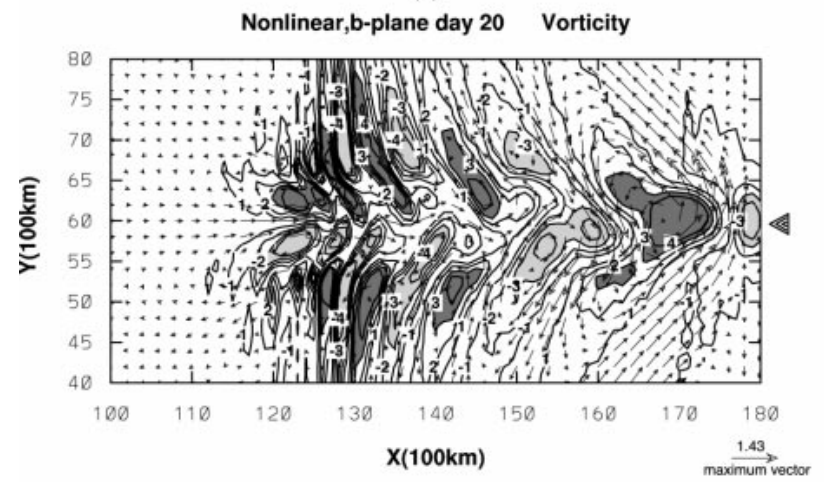

(d)
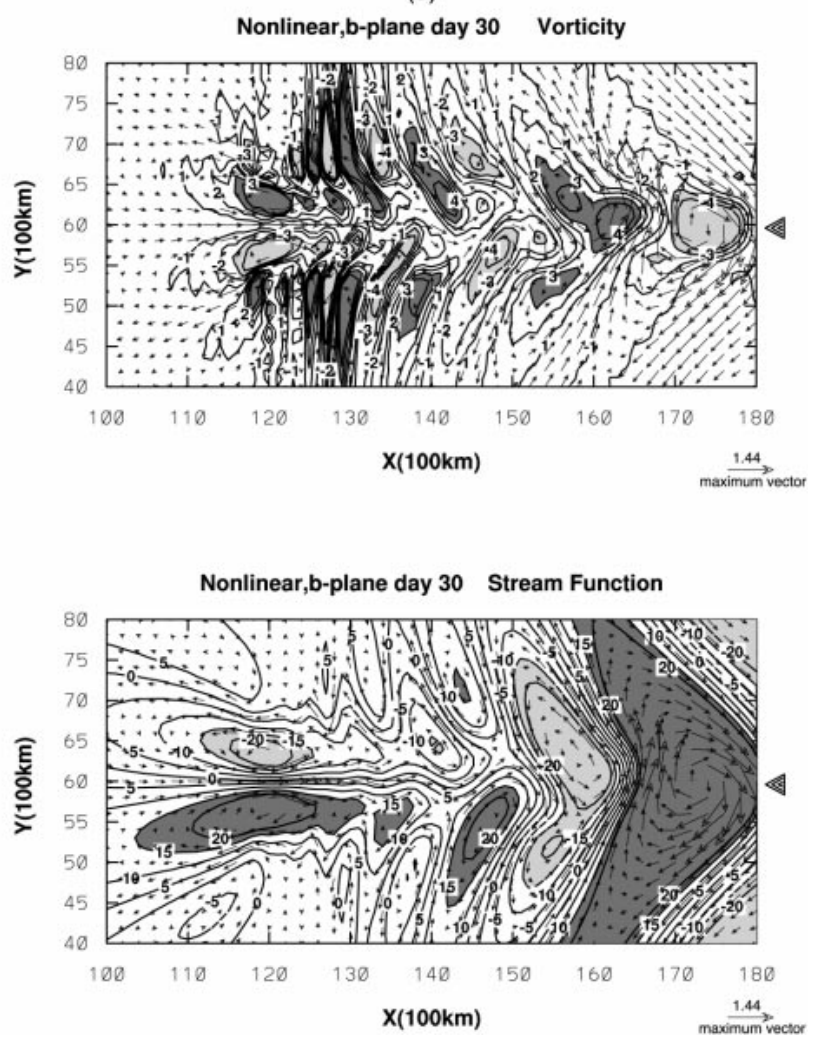

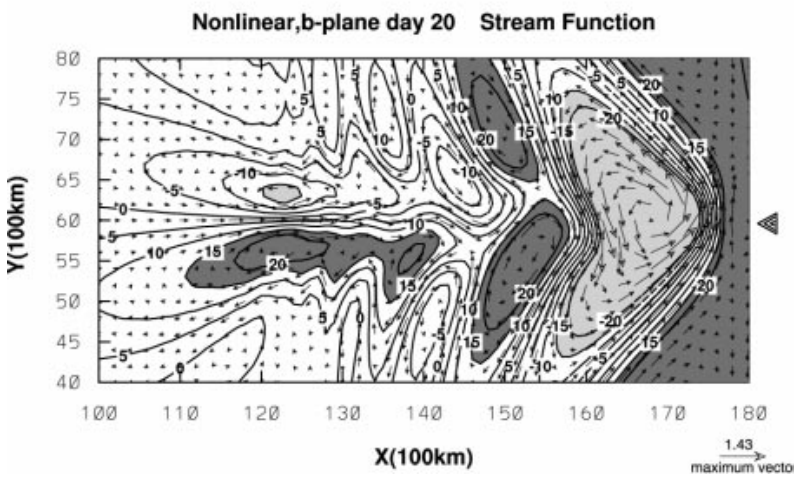

FIG. 5. (Continued)

of the confluence zone. The background flow as shown in Fig. 9, resembles an averaged $850-\mathrm{hPa}$ horizontal flow in the Northwest Pacific during periods when the northwestward-propagating disturbances are active (Lau

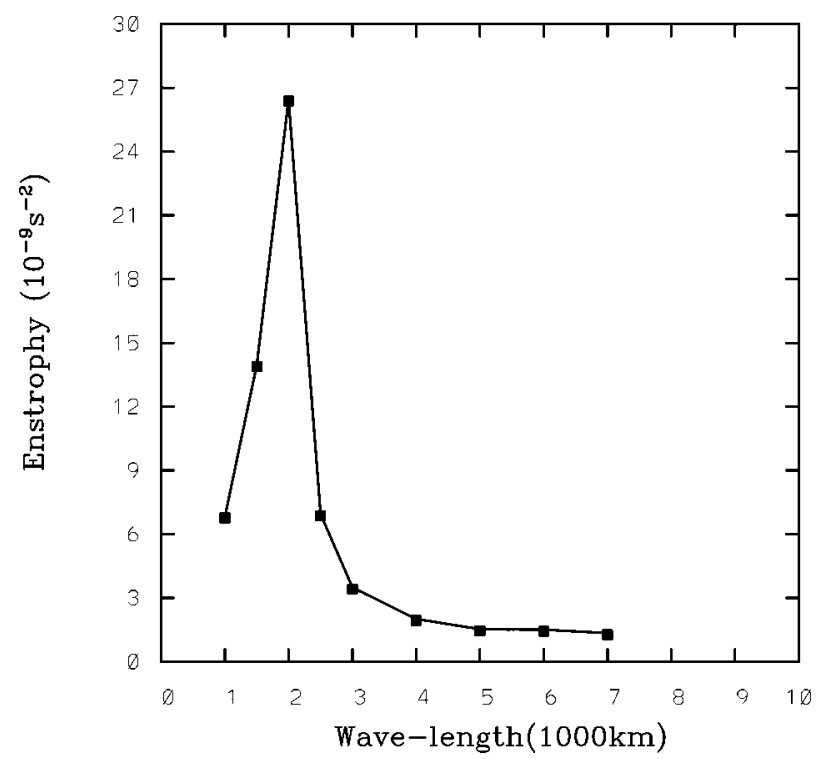

FIG. 6. The averaged enstrophy from days 140 to 200 as a function of forcing zonal wavelength for the nonlinear $\beta$-plane calculations. and Lau 1990, Fig. 17b). A Gaussian shape of convergence similar to the previous deformation basic flow experiments is applied to the center of the domain from day 15 to day 20, simulating a period of increased largescale convection. To examine the difference in the linear and nonlinear energy dispersion processes, we first conduct a linear calculation using a very large convergence value to boost the vorticity field. This is for the purpose of producing linear responses with amplitudes compatible to those of the nonlinear case. The peak convergence for the linear case is $-1.5 \times 10^{-5} \mathrm{~s}^{-1}$, which is about an order larger than the convergence used for the nonlinear case.

Figure 10 gives the wind vectors and the vorticity field for the linear $\beta$-plane calculation with a forcing zonal wavelength of $2500 \mathrm{~km}$ in the monsoonlike background flow experiment at (a) day 18, (b) day 22, and (c) day 26. The disturbance at day 18 results from the very large convergence imposed. Here a north-northwestward propagating disturbance is dispersing energy to the southeast toward the equatorial confluence zone. A series of elongated cyclonic and anticyclonic circulations result from the energy dispersion from days 18 to 22 . The circulations are elongated in the northeastsouthwest direction as expected from the linear Rossby energy dispersion theory. The spatial scale of the energy dispersion is contracted somewhat near the critical lon- 


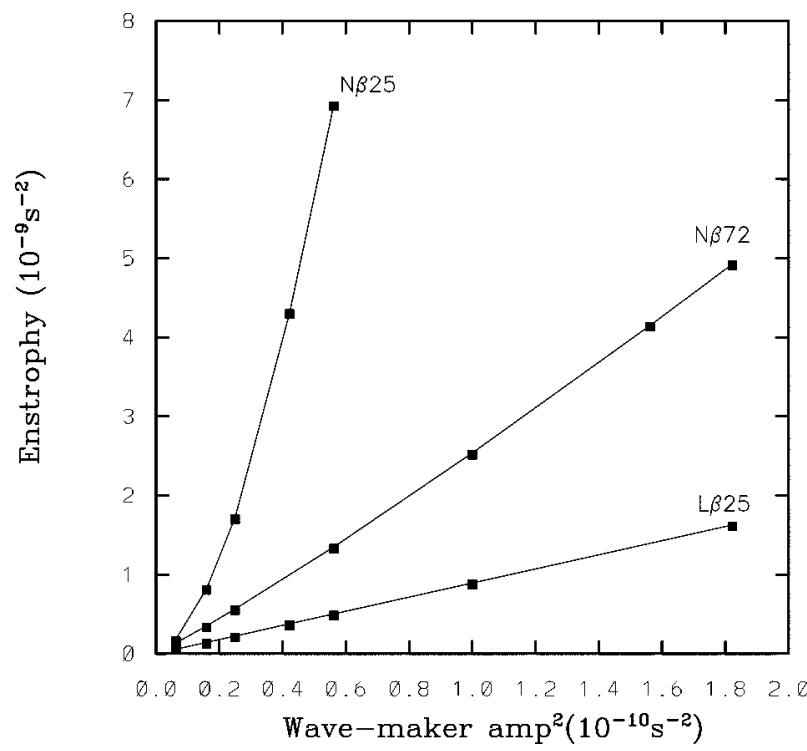

Fig. 7. The averaged enstrophy from days 140 to 200 as a function of forcing wave amplitude squared. The three curves are a nonlinear $\beta$-plane calculation with a forcing zonal wavelength $2500 \mathrm{~km}(\mathrm{~N} \beta 25)$, a nonlinear $\beta$-plane calculation with a forcing zonal wavelength 7200 $\mathrm{km}(\mathrm{N} \beta 72)$, and a linear $\beta$-plane calculation with a forcing zonal wavelength $2500 \mathrm{~km}(\mathrm{~L} \beta 25)$.

gitude. These stretched disturbance patterns clearly do not resemble observed tropical disturbances, which tend to have an aspect ratio closer to unity. In these cases additional effects, such as diabatic heating on the dis-

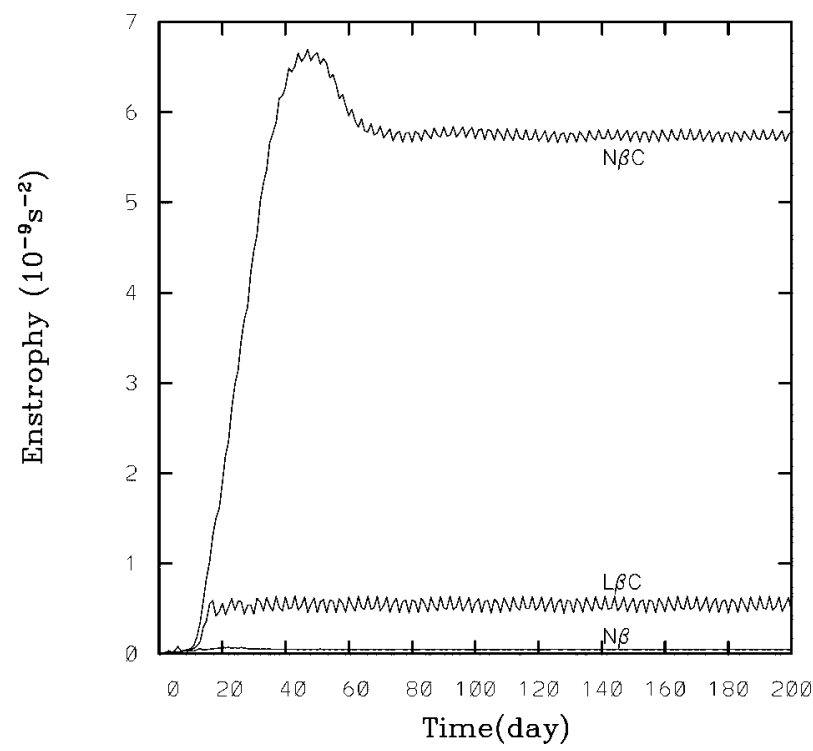

FIG. 8. The enstrophy as a function of time for the deformation background flow experiments. The three curves are a nonlinear $\beta$-plane calculation with an added convergence $(\mathrm{N} \beta C)$, a linear $\beta$-plane calculation with an added convergence ( $\mathrm{L} \beta \mathrm{C})$, and a nonlinear $\beta$-plane calculation without added convergence $(\mathrm{N} \beta)$. The forcing zonal wavelength is $2500 \mathrm{~km}$ for all cases. The added convergence is of a Gaussian profile with a $2500 \mathrm{~km} e$-folding distance in both $x$ and $y$ directions. The peak magnitude of convergence is $-4 \times 10^{-6} \mathrm{~s}^{-1}$.

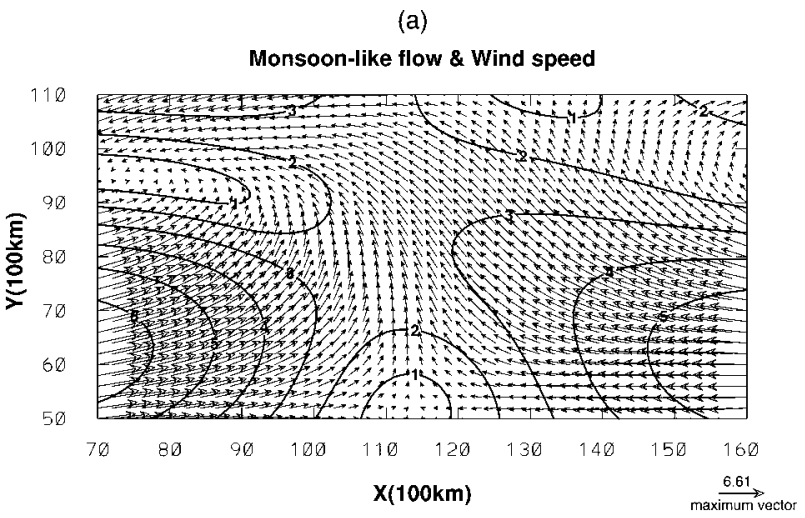

(b)

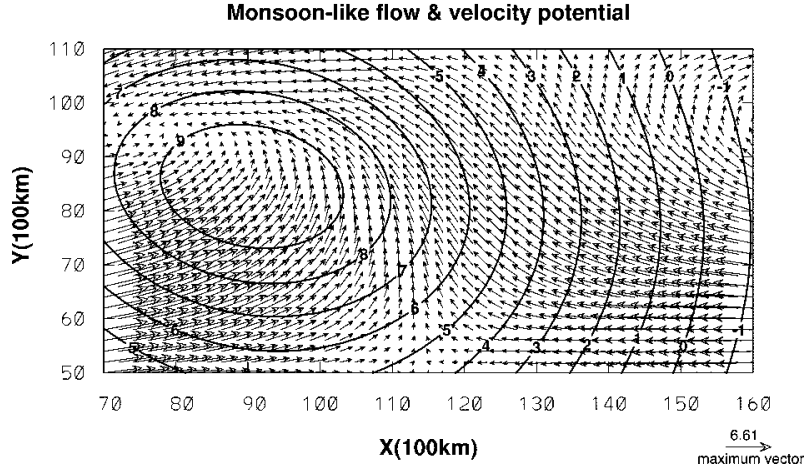

FIG. 9. Wind vectors of the monsoonlike background flow with isopleths of (a) wind speed $\left(\mathrm{m} \mathrm{s}^{-1}\right)$ and (b) velocity potential $\left(10^{-6}\right.$ $\left.\mathrm{m}^{2} \mathrm{~s}^{-1}\right)$. The peak background convergence is $-4 \times 10^{-6} \mathrm{~s}^{-1}$.

turbance scale, would be needed to hold the disturbance structure against dispersion.

Figure 11 shows the same integration results as Fig. 10 except that it is for the nonlinear $\beta$-plane calculation. Here we also see a north-northwestward propagating disturbance that is dispersing energy to the southeast toward the equatorial confluence zone, and a series of cyclonic and anticyclonic circulations resulting from the energy dispersion from days 18 to 22 . It is interesting to note that the horizontal pattern at day 22 (Fig. 11b) now does not show the unrealistic stretched structure. The disturbances here resemble the 6-10-day northwestward phasepropagating disturbance observed by Lau and Lau (1990) and by Chang et al. (1996). The formation of the disturbance near the critical longitude is due to three effects: the added convergence applied during days 15 to 20 , the scale contraction, and the nonlinear dynamics. The northwestward-propagating disturbance remains coherent and moves northwestward into higher latitudes (Fig. 11c). Thus, the nonlinear dynamics in the presence background convergence effect (i.e., Fig. 9b) can maintain the northwestward-propagating disturbance structure against dispersion, without the need of disturbance-scale diabatic heating or other organizing effects.

The dynamical basis for the coherent barotropic vortex can also be discussed in terms of the so-called $\beta$ 
(a)

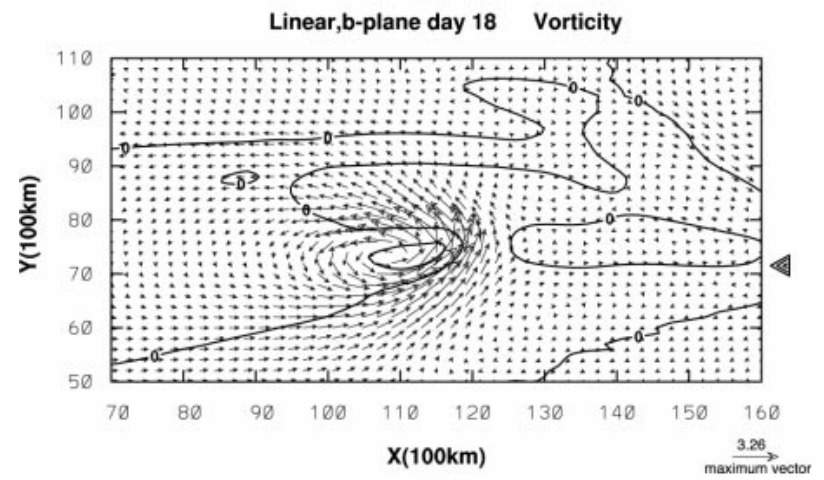

(c)

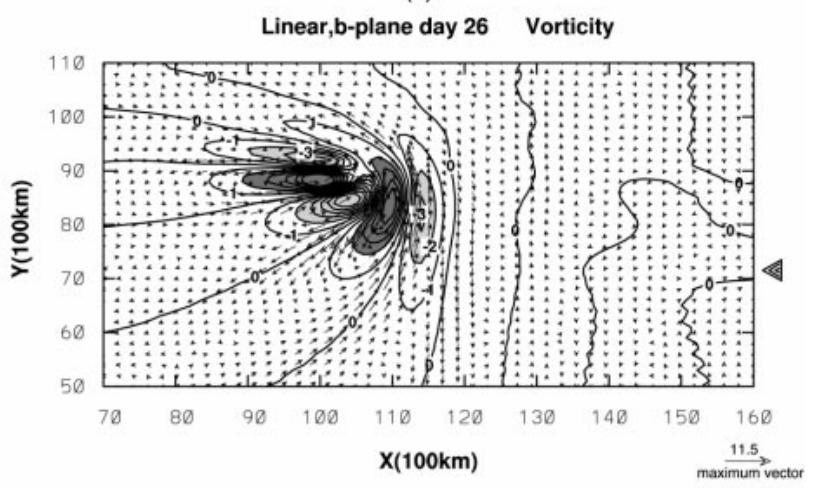

(b)

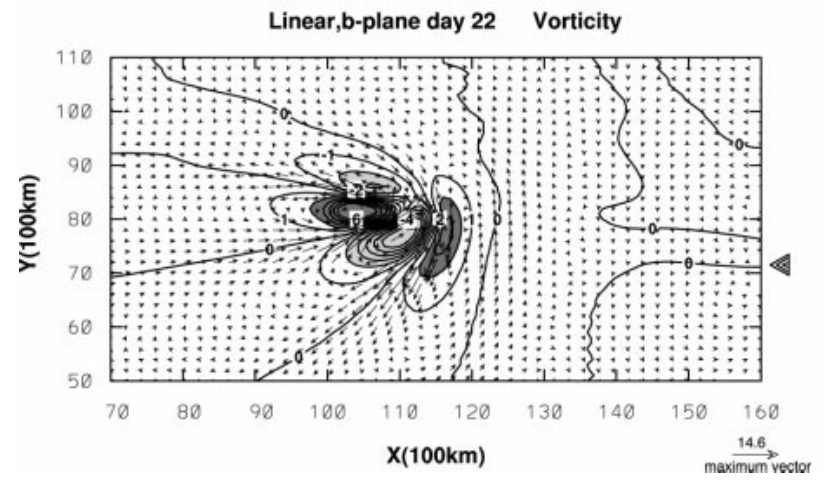

FIG. 10. Wind vectors and the vorticity field for the linear $\beta$-plane calculation with a forcing zonal wavelength $2500 \mathrm{~km}$ in the monsoonlike background flow experiment at (a) day 18 , (b) day 22 , and (c) day 26 . The vorticity isopleth interval is $10^{-5} \mathrm{~s}^{-1}$, with values $>2 \times$ $10^{-5} \mathrm{~s}^{-1}$ heavy-shaded and values $<-2 \times 10^{-5} \mathrm{~s}^{-1}$ light-shaded. The central latitude of east boundary forcing (right edge arrow) is at $Y=$ $7200 \mathrm{~km}$.

Rossby number (e.g., McWilliams and Flierl 1979). The $\beta$ Rossby number is the ratio of the nonlinear terms in the vorticity equation to the linear vortex Rossby wave dispersion term. The $\beta$ Rossby number in our application can be defined as

$$
R_{\beta}=\frac{V}{\beta r^{2}},
$$

where $V$ and $r$ are the vortex velocity scale and spatial scale, respectively. When this dimensionless parameter drops below unity, a stronger $\beta$ dispersion from the vortex is expected. When the number is of order of unity, the nonlinear effect is important and the vortex can hold itself against the dispersion. With the $r$ of the order 100 $\mathrm{km}$ in our calculations, it requires a $V$ of a few meters per second for the nonlinear effect to be important. The nonlinear dynamics thus are important for the coherent vortices in Fig. 11. The nonlinear dynamics of "dispersion-resistance" was also studied by Carr and Williams (1989) with the bounded Rankine vortex with zero basic-state vorticity gradient. Smith and Montgomery (1995) probably were the first to study the effects in the presence of a radially continuous vorticity field. Dritschel (1998) and Kuo et al. (1999) applied the dynamics in the context of the maintenance of elliptical vortices.
Due to the nonlinear interaction of the disturbance with the planetary vorticity gradient, the propagation speed is slightly faster than the advective speed by the background flow. This is consistent with the findings of Sobel and Bretherton (1999). A new disturbance forms at the south-southeast of the original disturbance in the confluence zone at day 26 (Fig. 11c). Since there is no convergence effect operating near the critical longitude after day 20, the growth of new disturbances cannot be due to the amplification of the incoming waves by the accumulation mechanism. In fact, we have performed the same experiment as in Fig. 11 without the continuous wave emanation after day 15 . The results (not shown) are similar to Fig. 11. Figure 11 shows that the successive formation of new disturbances is due to barotropic energy dispersion from the northwestward-propagating disturbance in the monsoonlike background flow. Figure 11c indicates the formation of the new vortex by the axisymmetrization dynamics (e.g., Melander et al. 1987; Guinn and Schubert 1993) from an elongated vortex sheet (i.e., Fig. 11b). Due to the asymmetry damping by the axisymmetrization or vortex stablization processes (e.g., Carr and Williams 1989; Smith and Montgomery 1995), the aspect ratio of the nonlinear disturbances is much closer to unity than the linear cas- 
(a)

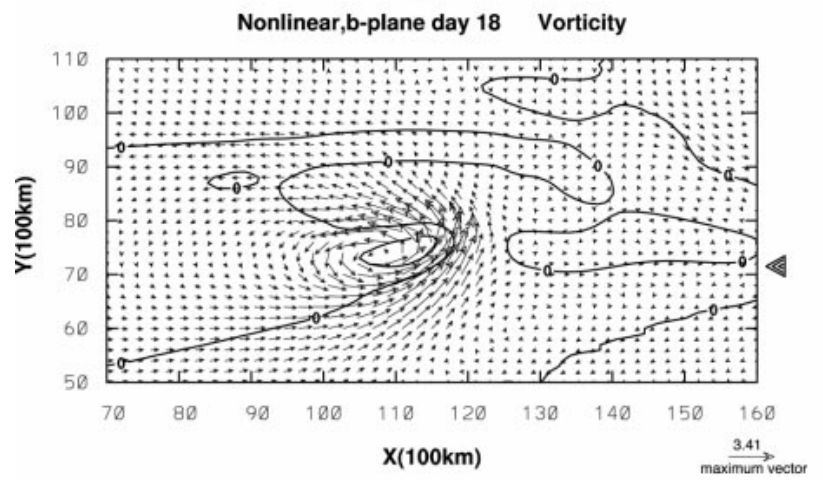

(b)

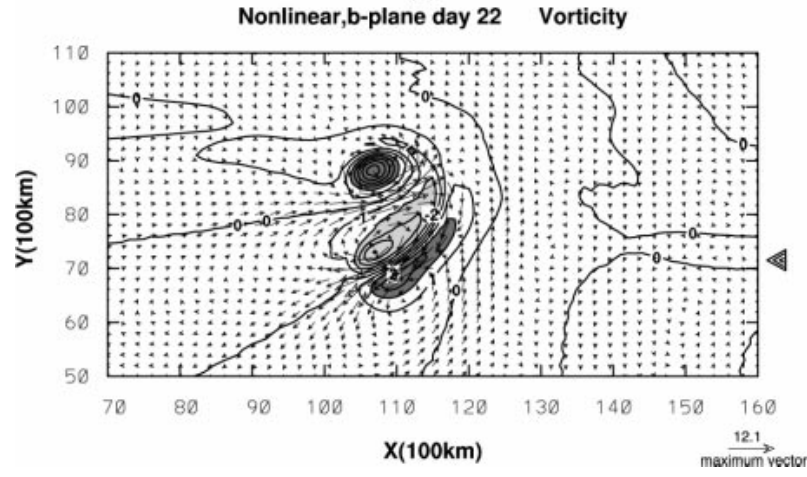

(c)

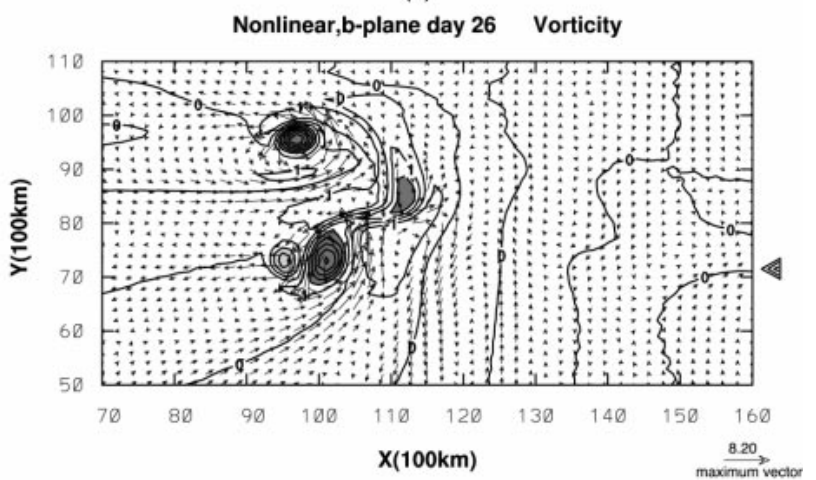

FIG. 11. Same as in Fig. 10 except for the nonlinear $\beta$-plane calculation.

es. The successive formation of new disturbances is about every 8 days, and the spatial scale between them is about $3000 \mathrm{~km}$. These values are in general agreement with the observational studies of Chang et al. (1996) and Sobel and Bretherton (1999).

The formation of new, trailing disturbances by the energy dispersion of the northwestward-propagating disturbance has been hypothesized by Chang et al. (1996) and Sobel and Bretherton (1999). To investigate the formation mechanism further, we consider some nonlinear initial value experiments. We place an initial vortex with the tangential wind defined as

$$
V=\frac{V_{m}}{2}\left[1-\cos \left(\pi \frac{r}{R_{m}}\right)\right], \quad 0 \leq r \leq 2 R_{m},
$$

where $V_{m}=12 \mathrm{~m} \mathrm{~s}^{-1}, R_{m}$ is the size parameter, and $r$ is the radius from the domain center. The vorticity field and wind vectors for the nonlinear initial value experiment in the monsoonlike background flow at (a) day 2, (b) day 4, (c) day 6, (d) day 8, and (e) day 10 are shown in Fig. 12. The size of the initial vortex is given by $R_{m}=500$ $\mathrm{km}$. We observe from Fig. 12 the northwestward propagation of the initial disturbance and the initiation of a new cyclonic disturbance slightly to the west of the critical longitude. The formation time, as well as the distance between the two vortices, is similar to the results from the forced experiment in Fig. 11. Figure 13 is similar to
Fig. 12 except that it does not contain the monsoonlike background flow. In this case, no new disturbance forms. Moreover, the energy dispersion in Fig. 13 occurs at a larger spatial scale and the aspect ratio of the cyclonic circulations is not close to unity. The nonlinearity is not important in this case due to the absence of the scale contraction, which depends on the zonally varying mean flow. Thus, the circulations are elongated in the northeast and southwest direction, similar to the results of the linear Rossby energy dispersion.

The above results suggest that the energy dispersion associated with a northwestward-propagating disturbance in a monsoonlike background flow can lead to the formation of a new disturbance southeast of the original disturbance. The energy dispersion in the presence of a monsoonlike background flow can be quite different from that without a background confluent flow. The difference lies in the scale contraction by the zonally varying background flow and the nonlinear effect, both of which are important for the initiation of tropical disturbances by the vortex axisymmetrization dynamics.

\section{Concluding remarks}

A nonlinear barotropic model is used to investigate the dynamics of energy dispersion and wave accumulation in zonally opposing background flows. When the 
(a)

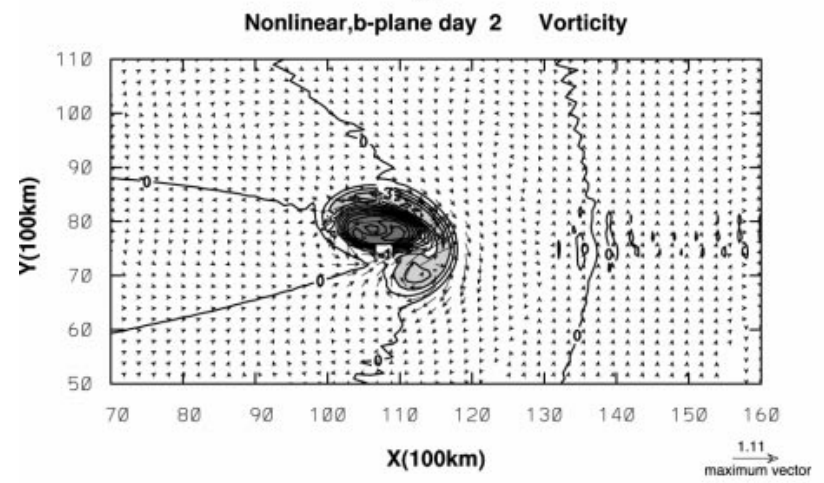

(b)

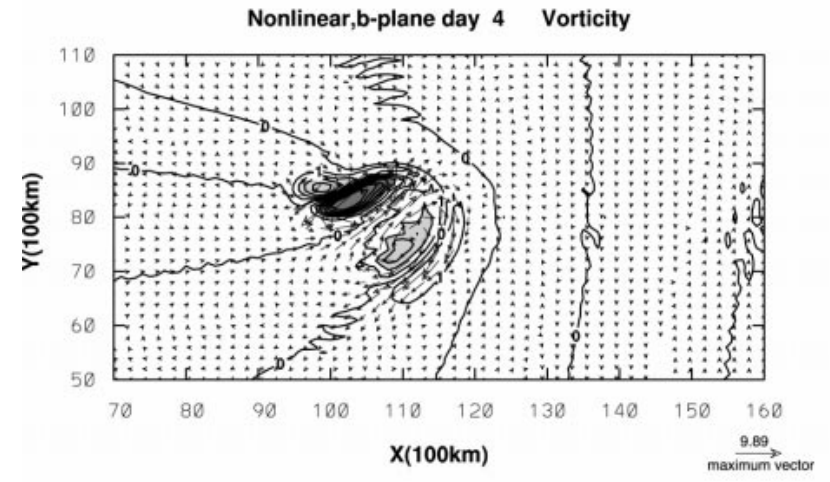

(c)

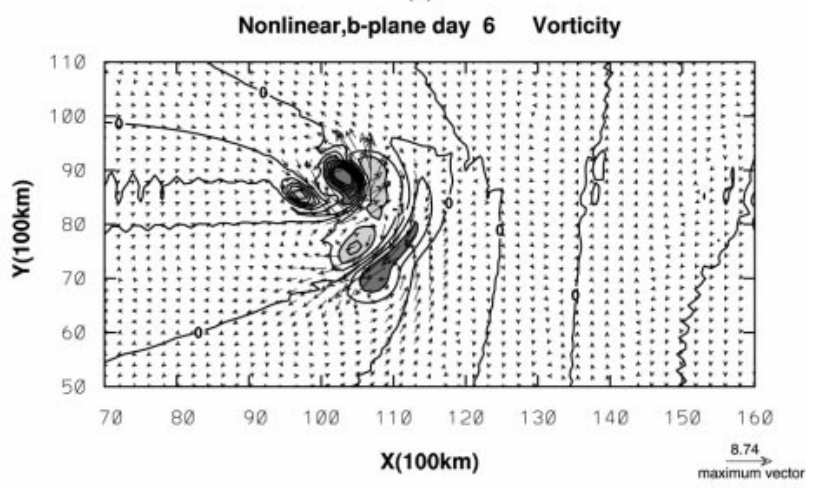

(d)

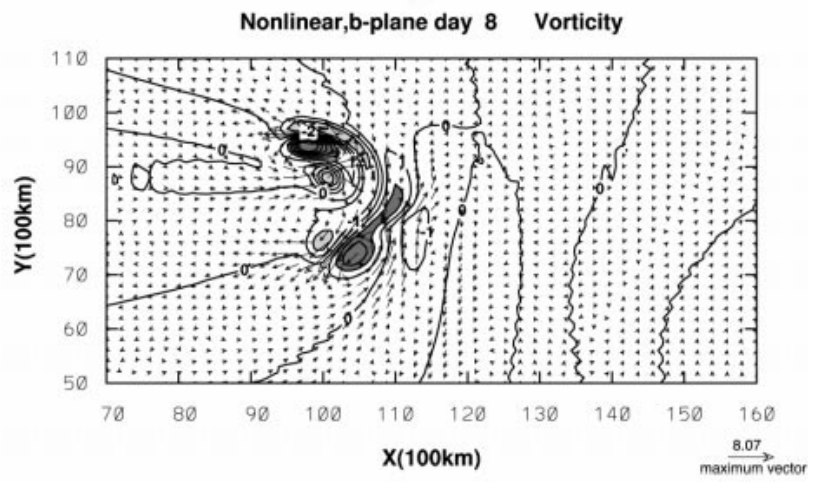

(e)

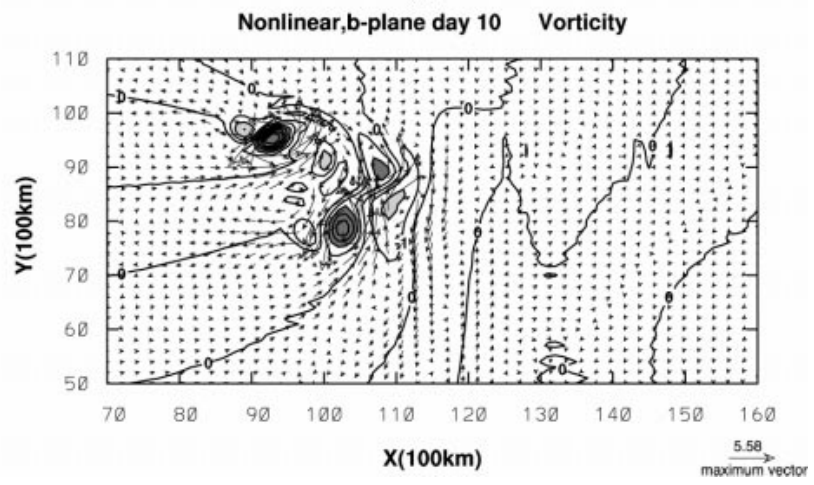

FIG. 12. The vorticity field for the nonlinear initial value experiment with the monsoonlike background flow at (a) day 2, (b) day 4, (c) day 6, (d) day 8, and (e) day 10. The size of the initial vortex is given by $R_{m}=500 \mathrm{~km}$. The isopleth interval is $10^{-5} \mathrm{~s}^{-1}$, with values $>2$ $\times 10^{-5} \mathrm{~s}^{-1}$ heavy-shaded and values $<-2 \times 10^{-5} \mathrm{~s}^{-1}$ light-shaded.

model is applied to a confluent zonal flow resembling the tropical Northwest Pacific summer monsoon, formation of tropical vortex disturbances occurs as a result of the interaction of propagating short Rossby waves with the monsoonlike background flow. The large-scale convergence, the scale contraction, and the nonlinear dynamics on the $\beta$ plane provide the essential mechanisms for the initial development of tropical disturbances through nonlinear energy/enstrophy accumulation in the confluence zone. The development can be maintained with a continuous Rossby wave emanation upstream. The largest accumulation occurs when the emanating zonal wavelength is around $2000 \mathrm{~km}$. This preferred wavelength emerges as a compromise between longer waves that experience less scale contraction and nonlinear effects and shorter waves that cannot hold a coherent structure against dispersive effects. With the monsoonlike mean flow the nonlinear process further allows the formation of a new vortex disturbance through energy dispersion by a previous northwestwardpropagating disturbance.

There are significant differences between the nonlin- 
(a)

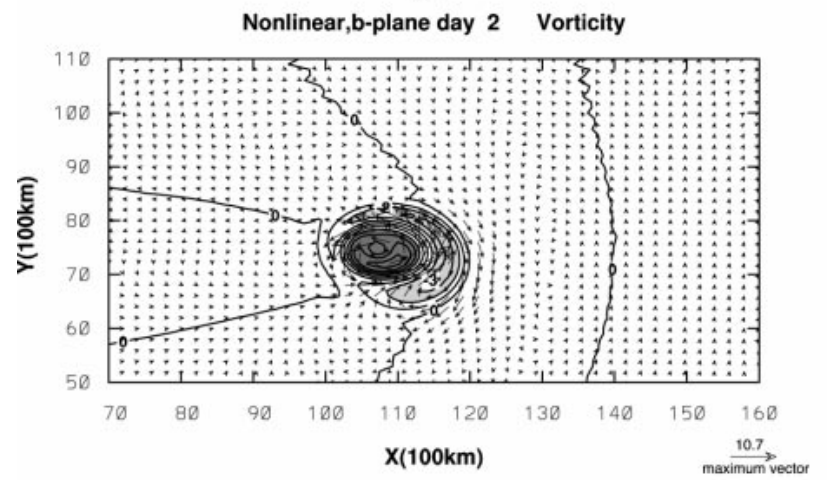

(c)

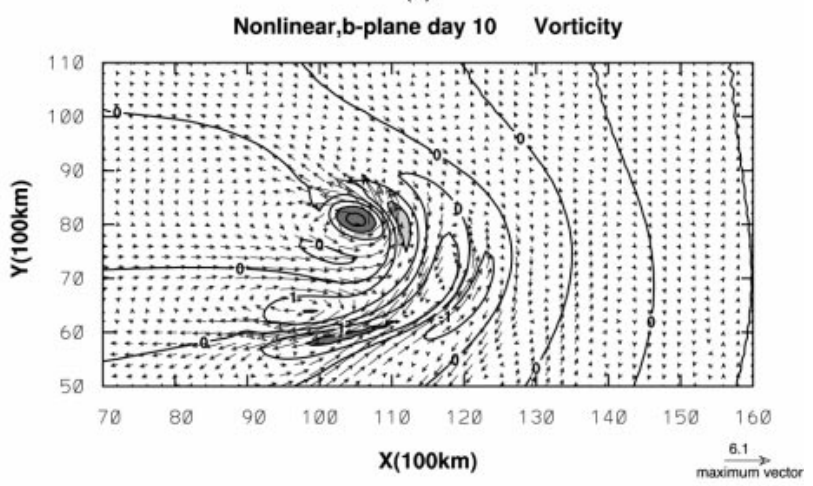

(b)

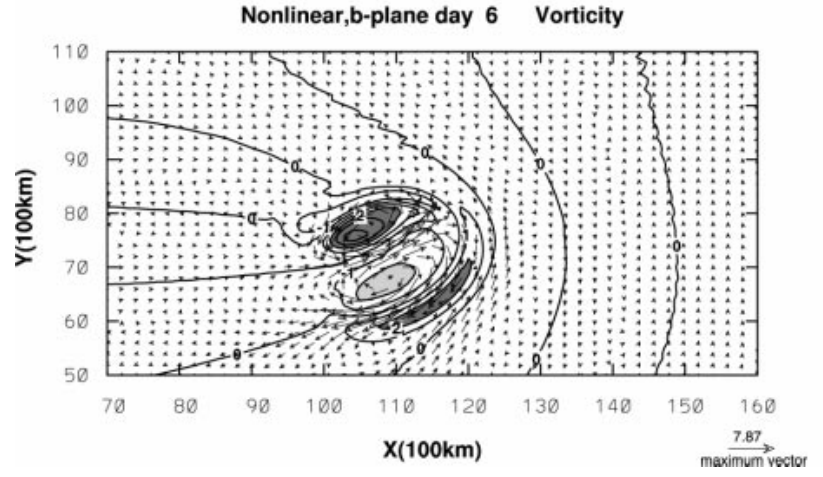

FIG. 13. Similar to Fig. 12 except without the monsoonlike background flow and at (a) day 2, (b) day 6, and (c) day 10.

ear energy/enstrophy accumulation mechanism and the linear theories. In the linear theories (e.g., Webster and Chang 1988; Holland 1995; Sobel and Bretherton 1999) the convergence of the mean zonal advection causes a slowdown of the Doppler-shifted group velocity that leads to the energy accumulation. However, Farrell and Watterson (1985) have shown that the increase of zonal wavenumber due to scale contraction will result in an eventual loss of wave energy to the mean flow via the Reynold's stress. Thus, without the presence of other forcing processes such as disturbance-scale diabatic heating, the disturbances will decay under the linear theories. On the other hand, after the initial convergence of the Doppler-shifted group velocity, in the nonlinear theory the vorticity gradient in the waves is sharpened by the background flow and the nonlinear dynamics becomes dominant. As a result, disturbance asymmetries with respect to the central latitude develop due to the nonlinear interaction of Rossby waves with the planetary vorticity gradient. This aspect is similar to the argument made by Shapiro (1977) on the formation of tropical storms from easterly waves. Shapiro (1977) viewed the sharpening of the scale of the wave itself over its own scale [as measured by $\epsilon^{\prime}=(d k / d x) / k^{2}$ with $k$ wavenumber] as the key for the wavelike (linear) structure to become a stormlike (nonlinear) structure. In our model this development leads to a pair of vorticity centers that straddles the central latitude with a cyclone in the north and an anticyclone in the south, and an elongated, weak westerly flow along the central latitude near the critical longitude. This elongated westerly flow, which possesses a smaller zonal wavenumber as compared to that in the linear case, reverses the sign of the Reynold's stress and causes the energy to grow near the critical longitude, leading to the intensification of disturbances.

In the situation that the background flow field allows a confluence effect but no convergence effect, a largescale convergence comparable to the magnitude of the frictional effect is required in the nonlinear energy/enstrophy accumulation process near the critical longitude. In addition to overcoming the friction damping, this large-scale convergence is to assume the essential role of the initial convergence of the mean zonal advection. It presumably is produced by the background convective forcing that is associated with monsoon heating. Again, diabatic effects on the disturbance scale are not involved in our argument. The nonlinear dynamics can hold the disturbance in a coherent fashion against dispersion without disturbance-scale diabatic effects.

When a mean flow with a flow reversal similar to that of the low-level Northwest Pacific summer monsoon is present, the energy dispersion in the presence of scale contraction leads to the formation of new dis- 
turbances in the confluence zone by the vortex axisymmetrization dynamics (the nonlinear process in which the vortex tends to damp asymmetries in order to maintain the symmetric circulation). In our results the successive formation of new disturbances has a period of approximately 8 days and a spatial scale of about 3000 $\mathrm{km}$, which are in general agreement with observations in the Northwest Pacific (Lau and Lau 1990; Chang et al 1996). This vortex formation is not possible using the linear theory of Rossby wave energy dispersion. [Holland (1995) did find new vortex formation from the barotropic energy dispersion without the background confluent flow. However, his baroclinic model results are obtained with a diabatic heating that is proportional to the low-level cyclonic vorticity.] Thus, our results suggest the possibility that the nonlinear processes in an opposing mean zonal flow can lead to vortex development from tropical waves without disturbancescale diabatic heating. This process may explain the observed northwestward-propagating series of synopticscale tropical depressions in the low-level monsoon confluence zone (e.g., Lau and Lau 1990; Chang et al. 1996; Sobel and Bretherton 1999), in which the growth of a new disturbance was often observed to occur to the southeast of the previous disturbance.

Although our calculations contain no mean relative vorticity (i.e., $\bar{\zeta}=0$ ), our discussion can shed some light on the situation when mean relative vorticity is present. If the cyclonic vorticity center is situated at low latitudes, it is possible to have some cancelation of the $(d \bar{\zeta} / d y) v^{\prime}$ term and the $\beta v^{\prime}$ term in (2.1). In such a case, the process of energy/enstrophy accumulation and the energy dispersion effects would be weakened in the confluence zone. On the other hand, the elongated circulations due to the energy dispersion can interact nonlinearly with the background shear (vorticity). An example of the nonlinear interaction can be found in Ferreira and Schubert (1999). They showed that in the presence of cyclonic or weak anticyclonic shear, the trough to the east of tropical cyclone may broaden, resulting in the formation of an intense Tropical Upper Tropospheric Trough cell that has a strong signature in the potential vorticity field. Thus, it is possible that in addition to the scale contraction the energy dispersion in the presence of cyclonic background shear may also help the formation of a new coherent vortex.

It has been observed that the tropical 4-5 day wave disturbances tend to have a vertically propagating structure in the central Pacific near the dateline and deep, warm core structure in the western Pacific west of $160^{\circ} \mathrm{E}$ (e.g., Chang et al. 1970). Even at the same longitude, the former type dominates in La Niña years when the easterly trades prevail and the latter type dominates in El Niño years when westerly winds penetrate anomalously eastward (Chang and Miller 1977; Takayabu and Nitta 1993). The barotropic mechanisms identified in this study may play a role in the development of the deep, warm-core tropical depressions in the western $\mathrm{Pa}$ cific from waves propagating from the east.

In a study of tropical cyclone development in the equatorial western Pacific during the northern winter monsoon of 1996/97, Taylor (1998) documented several cases in which the development appears to be a result of the interaction of the eastward-propagating MaddenJulian oscillation (MJO) and a synoptic easterly wave from the central Pacific. Since the MJO possesses a lowlevel westerly maximum near the equator, a region of confluent and convergent flow can be produced when the westerly flow meets the easterly flow at its forward edge. There is little doubt that diabatic heating is very important in the westerly surge and the tropical depression formation, but in these cases the nonlinear wave accumulation mechanism may also contribute. Maloney and Hartmann (2000) showed that the eastern Pacific is subject to a substantial MJO that has a major impact on the frequency of cyclogenesis and the number of hurricane-strength disturbances; both are much higher during active MJO periods. Molinari and Vollaro (2000) suggested that the growth of unstable waves due to the sign reversal of the background meridional potential vorticity gradient in the eastern Pacific is strongest during the active MJO. It is possible that the enhanced westerlies ahead of MJO and the background easterlies also provide a favorable basic state for the nonlinear wave accumulation mechanism.

The present results suggest the importance of the background confluent flow, scale contraction of short Rossby waves, and nonlinear dynamics for the successive formation of tropical disturbances. In addition to these barotropic effects, other factors such as sea surface temperature, convective activity, and local Walker and Hadley circulations will also modulate the dynamics of the confluence zone. Further studies of these effects will require the use of a baroclinic primitive equation model.

Acknowledgments. This research was supported by the National Science Foundation under Grant ATM9525755. HCK is also supported by the National Research Council of Taiwan under Grants NSC88-2111M002-017 AP1 and NSC88-2625-Z002-029. We would like to thank Drs. L. E. Carr III and Ching-Hwang Liu for producing the satellite picture used in Fig. 1. We would also like to thank Dr. A. Sobel and two anonymous reviewers for useful comments in the paper.

\section{REFERENCES}

Carr, L. E., and R. T. Williams, 1989: Barotropic vortex stability to perturbations from axisymmetry. J. Atmos. Sci., 46, 3177-3191.

Chan, C. L., and R. T. Williams, 1987: Analytical and numerical studies of the beta-effect in tropical cyclone motion. Part I: Zero mean flow. J. Atmos. Sci., 44, 1257-1265.

Chang, C.-P., and C. R. Miller III, 1977: Comparison of easterly waves in the tropical Pacific during two contrasting periods of seasurface temperature anomalies. J. Atmos. Sci., 34, 615-628.

, V. F. Morris, and J. M. Wallace, 1970: A statistical study of 
easterly waves in the western Pacific July-December 1964. J. Atmos. Sci., 27, 195-201.

_ J.-M. Chen, P. A. Harr, and L. E. Carr, 1996: Northwestwardpropagating wave patterns over the tropical western North Pacific during summer. Mon. Wea. Rev., 124, 2245-2266.

Chang, H.-R., and P. J. Webster, 1990: Energy accumulation and emanation at low latitudes. Part II: Nonlinear response to strong episodic equatorial forcing. J. Atmos. Sci., 47, 2624-2644.

— , and 1995: Energy accumulation and emanation at low latitudes. Part III: Forward and backward accumulation. J. Atmos. Sci., 52, 2384-2403.

Davidson, N. E., and H. H. Hendon, 1989: Downstream development in the Southern Hemisphere monsoon during FGGE/WMONEX. Mon. Wea. Rev., 117, 1458-1470.

Dritschel, D. G., 1998: On the persistence of non-axisymmetric vortices in inviscid two-dimensional flows. J. Fluid Mech., 371, 141-155.

Farrell, B., and I. Watterson, 1985: Rossby waves in opposing currents. J. Atmos. Sci., 42, 1746-1756.

Ferreira, R. N., and W. H. Schubert, 1999: The role of tropical cyclones in the formation of tropical upper-tropospheric troughs. J. Atmos. Sci., 56, 2891-2907.

Guinn, T., and W. H. Schubert, 1993: Hurricane spiral bands. J. Atmos. Sci., 50, 3380-3404.

Holland, G., 1995: Scale interaction in the western Pacific monsoon. Meteor. Atmos. Phys., 56, 57-79.

Kuo, H. C., R. T. Williams, and J. H. Chen, 1999: A possible mechanism for the eye rotation of typhoon Herb. J. Atmos. Sci., 56, $1659-1673$.

Lau, K.-H., and N.-C. Lau, 1990: Observed structure and propagation characteristics of tropical summertime synoptic scale disturbance. Mon. Wea. Rev., 118, 1888-1913.

, and — 1992: The energetics and propagation dynamics of tropical summertime synoptic scale disturbances. Mon. Wea. Rev., 120, 2523-2539.
Maloney, E. D., and D. L. Hartmann, 2000: Modulation of eastern North Pacific hurricanes by the Madden-Julian oscillation. $J$. Climate, 13, 1451-1460.

McWilliams, J. C., and G. R. Flierl, 1979: On the evolution of isolated, nonlinear vortices. J. Phys. Oceanogr., 9, 1183-1206.

Melander, M. V., J. C. McWilliams, and N. J. Zabusky, 1987: Axisymmetrization and vorticity-gradient intensification of an isolated two-dimensional vortex through filamentation. J. Fluid Mech., 178, 137-159.

Molinari, J., and D. Volaro, 2000: Planetary and synoptic-scale influences on eastern Pacific tropical cyclogenesis. Mon. Wea. Rev., 128, 3296-3307.

Shapiro, L. J., 1977: Tropical storm formation from easterly waves: A criterion for development. J. Atmos. Sci., 34, 1007-1021.

— 1978: The vorticity budget of a composite African tropical wave disturbance. Mon. Wea. Rev., 106, 806-817.

Smith, G. B., and M. T. Montgomery, 1995: Vortex axisymmetrization: Dependence on azimuthal wavenumber or asymmetric radial structure changes. Quart. J. Roy. Meteor. Soc., 121, 16151650.

Sobel, A. H., and C. S. Bretherton, 1999: Development of synopticscale disturbances over the summertime tropical northwest Pacific. J. Atmos. Sci., 56, 3106-3127.

Stevens, D. E., 1979: Vorticity, momentum and divergence budgets of synoptic-scale wave disturbances in the tropical eastern Atlantic. Mon. Wea. Rev., 107, 535-550.

Takayabu, Y. N., and T. Nitta, 1993: 3-5 day-period disturbances coupled with convection over the tropical Pacific Ocean. J. Meteor. Soc. Japan, 71, 221-246.

Taylor, S. C., 1998: Interaction of large-scale tropical motion systems during the 1996-1997 Australian monsoon. M.S. thesis, Naval Postgraduate School, Department of Meteorology, 107 pp.

Webster, P. J., and H.-R. Chang, 1988: Equatorial energy accumulation and emanation regions: Impact of a zonally varying basic state. J. Atmos. Sci., 45, 803-829. 